\title{
Monopoly Quality Degradation and Regulation in Cable Television
}

\author{
Gregory S. Crawford University of Arizona \\ Matthew Shum Johns Hopkins University
}

\begin{abstract}
Using an empirical framework based on the Mussa-Rosen model of monopoly quality choice, we calculate the degree of quality degradation in cable television markets and the impact of regulation on those choices. We find lower bounds of quality degradation ranging from 11 to 45 percent of offered service qualities. Furthermore, cable operators in markets with local regulatory oversight offer significantly higher quality, less degradation, and greater quality per dollar, despite higher prices.
\end{abstract}

\section{Introduction}

In many markets, firms choose not only the prices but also the qualities of their products. In many cases, this is the primary dimension on which firms compete, as in pharmaceutical, media, and professional services and many high-technology markets. Theorists have long recognized that in the presence of imperfect competition, offered qualities can be distorted from the social optimum because firms equate private instead of social marginal benefits and marginal costs (Dixit and Stiglitz 1977; Spence 1980). This induces a welfare loss analogous to that from price distortions. Indeed, aspects of a firm's product offerings, and not pricing, have been the focus of recent highly contested antitrust cases (for example, Microsoft, GE/Honeywell).

The tendency of firms with market power to distort quality has been most clearly formulated in the monopoly nonlinear pricing literature, in which it is shown that the firm's products suffer from quality degradation (Mussa and Rosen 1978; Maskin and Riley 1984). Because products of different qualities are substitutes, a monopolist cannot simultaneously offer each consumer his or her efficient quality and also extract his or her full surplus, even with a fully nonlinear

We would like to thank Gary Biglaiser, Silke Januszewski, Eugenio Miravete, and seminar participants at Northwestern University, the University of California, Los Angeles, the National Bureau of Economic Research 2002 winter program meeting, the Society for Economic Dynamics 2002 meeting, the 2004 Kiel-Munich Workshop on Network Industries, and the 2004 Centre for Economic Policy Research conference Competition in the New Economy for helpful comments.

[Journal of Law and Economics, vol. 50 (February 2007)]

(C) 2007 by The University of Chicago. All rights reserved. 0022-2186/2007/5001-0007\$10.00 
tariff. Instead, under standard assumptions, quality for all but consumers with the highest tastes for quality is distorted downward. Furthermore, consumers with low preferences for quality may be excluded entirely from the market. Regulation, by either minimum quality standards or price caps, generally reduces distortions but can have ambiguous effects on prices and welfare (Besanko, Donnenfeld, and White 1987, 1988).

Despite the widespread acknowledgment of the potential for quality degradation, measures of its extent and implications for outcomes in real-world markets are few. In this paper, we analyze quality degradation in a market long thought subject to its effects: the cable television industry. To do so, we introduce an empirical framework based on the Mussa-Rosen model that exploits the optimality conditions for the monopolist's quality choice problem to recover measures of the quality of the monopolist's offerings. This permits us to directly measure how much cable monopolies degrade quality relative to a competitive alternative. It also allows us to measure the impact of local regulatory oversight on ameliorating monopoly quality distortion.

We present two main results. First, we find evidence of substantial quality degradation in the cable television industry across a variety of specifications. While some firms offer two or three goods, most offer just a single product quality. Furthermore, offered qualities are at least 11.1 percent and 30.3 percent less in three-good markets and 44.7 percent less in two-good markets than what would be provided in a competitive market offering the same number of goods.

Second, we find that local regulatory oversight-in the form of certification by the local franchise authority to cap cable prices-has important ameliorative effects. Systems in franchise areas where the local franchise authority was certified offer an estimated 25.1 percent more services, 24.1 percent higher quality for low- and medium-quality goods (where offered), and greater quality per dollar to consumers despite higher prices. These results are consistent with the impact of minimum quality standards and could be of significant interest to policy makers concerned about the effectiveness of past regulatory interventions in the industry but troubled by continued growth in cable prices. ${ }^{1}$

The rest of this paper is organized as follows. In Section 2, we survey the canonical Mussa and Rosen (1978) model of monopoly quality choice that forms the foundation of the empirical analysis. We also present extensions to this model developed by Besanko, Donnenfeld, and White (1988) to allow for quality choice in the presence of regulation. In Section 3, we describe the cable television industry and discuss its suitability for this empirical analysis, followed in Section 4 by the empirical model and algorithm for recovering quality measures. Section 5 presents the results, and Section 6 concludes.

\footnotetext{
${ }^{1}$ The most recent report on cable prices by the Federal Communications Commission (FCC) found prices increased by 5.4 percent for the 12 months ending January 1, 2004, slightly less than the 5-year compound annual increase of 7.5 percent from 1998 to 2003 and far higher than the 1.5 percent increase in the Consumer Price Index over the same period (FCC 2005a).
} 


\section{The Incentives to Degrade Quality}

In this section, we discuss the quality degradation result from the theory of monopoly nonlinear pricing using a simple, two-type version of the model of Mussa and Rosen (1978). ${ }^{2}$ Consider a monopolist selling two goods, $q_{1}$ and $q_{2}$, whose qualities can be freely varied over $Q=[0, \bar{Q}]$. Consumers are assumed to be differentiated by a type parameter that takes on three distinct values, $t_{0}$, $t_{1}$, and $t_{2}\left(t_{0}<t_{1}<t_{2}\right)$, with respective probabilities $f_{i}$ (with $\left.f_{0}+f_{1}+f_{2}=1\right)$ and associated cumulative distribution function $F_{k} \equiv \sum_{j=0}^{k} f_{i}$. Type $0, t_{0}$, is included to allow for the possibility that some consumers prefer not to purchase either of the firm's products. ${ }^{3}$ For convenience, we assume the hazard function for the type distribution, $f_{i} /\left(1-F_{i}\right)$, is increasing in $i^{4}{ }^{4}$ The monopolist is assumed to be able to offer a nonlinear tariff specifying a different total price per quality variant offered, $P_{1}$ and $P_{2}$. The firm knows the distribution of types in the population and selects the tariff that maximizes its expected profit (with the expectation taken over consumer types).

Consumer preferences are assumed to be quasi-linear in money, $u_{i} \equiv u(q$, $\left.t_{i}\right)=v\left(q, t_{i}\right)-P(q)$. A consumer of type $t_{i}$ is assumed to choose that bundle, $q_{i}$, which maximizes his or her utility, so that

$$
q_{i} \equiv \underset{q \in\left\{q_{1}, q_{2}\right\}}{\arg \max } u\left(q, t_{i}\right), \quad i=1,2 .
$$

Furthermore, given that no consumer can be forced to participate in the contract, the monopolist's choice of qualities and prices must be such that the consumer voluntarily chooses to accept the contract, which requires

$$
u\left(q_{i}, t_{i}\right) \geq 0, \quad i=1,2 .
$$

Equations (1) and (2) are the incentive compatibility (hereafter IC) and individual rationality (IR) constraints.

The firm's optimization problem is then to maximize expected profits,

$$
\max _{P(q)} E[\pi]=\sum_{i=1}^{2} f_{i}\left[P\left(q_{i}\right)-C\left(q_{i}\right)\right],
$$

subject to optimal behavior by consumers, as encompassed in the IC and IR

${ }^{2}$ Since the derivations in this section are standard, we omit a number of technical details; see, for example, Laffont and Tirole (1993, chap. 2) for complete details. Furthermore, the Mussa-Rosen model has recently been extended by Rochet and Stole (2002) to allow households random private values for the outside option, with interesting implications for the extent of and patterns in quality degradation. We explore the differing implications of these models in ongoing work (Crawford and Shum 2005) and note that the results we present here are conditional on our assumed form for household preferences.

${ }^{3}$ This "outside type" is generally not included in the typical theoretical exposition. We include it here to facilitate empirical implementation of the model, in which there are always some consumers who purchase the "outside good."

${ }^{4}$ This rules out bunching of types at a single quality variant. Wilson (1993, chap. 8.1) presents a detailed discussion of the conditions under which this assumption is likely to be violated. 
constraints. The term $C\left(q_{i}\right)$ is the firm's cost function, which is assumed to be purely additive across consumers. ${ }^{5}$ Define the total surplus function $S\left(q, t_{i}\right) \equiv$ $v\left(q, t_{i}\right)-C(q)$. Using a common trick from the screening literature, we can rewrite profits as the difference between the total and consumer surplus:

$$
\max _{u(q)} E[\pi]=\sum_{i=1}^{2} f_{i}\left[S\left(q_{i}, t_{i}\right)-u\left(q_{i}, t_{i}\right)\right] .
$$

In this reformulated problem, the monopolist solves for the optimal utility quality schedule and determines optimal prices (given utilities) from the binding IC constraints. Under standard assumptions, we can use the IC constraint to rewrite the objective function, which yields

$$
\max _{q_{1}, q_{2}, u_{1}} E[\pi]=f_{1}\left[S\left(q_{1}, t_{1}\right)-u_{1}\right]+f_{2}\left\{S\left(q_{2}, t_{2}\right)-\left[v\left(q_{1}, t_{2}\right)-v\left(q_{1}, t_{1}\right)\right]-u_{1}\right\} .
$$

This problem is solved by setting the utility of the lowest type to zero, $u_{1}=0$, and maximizing the resulting unconstrained objective function with respect to $q_{1}$ and $q_{2}$. The corresponding first-order conditions are

$$
S_{q}\left(q_{1}, t_{1}\right)=\frac{1-F_{1}}{f_{1}}\left[v_{q}\left(q_{1}, t_{2}\right)-v_{q}\left(q_{1}, t_{1}\right)\right] \quad \text { and } \quad S_{q}\left(q_{2}, t_{2}\right)=0
$$

where $v_{q} \equiv \partial v / \partial q$. Quality degradation for the low type $(i=1)$ is visible from equation (6). The socially optimal quality for each type, denoted $q_{i}^{\star *}$, is that which sets the derivative of the total surplus function to zero, $S_{q}\left(q, t_{i}\right)=0$. In equation (6), however, we see that $q_{1}$ is chosen so that $S_{q}\left(q, t_{1}\right)>0$, which implies that $q_{1}^{*}<q_{1}^{\star *}$ : quality is degraded to low types. However, there is no degradation at the top for the higher type $t_{2}$. Given optimal qualities from equation (6), optimal prices fall out naturally from the IR and IC constraints. Since $u_{1}=0$, $p_{1}^{\star}=v\left(q_{1}^{\star}, t_{1}\right)$ and $p_{2}^{\star}=v\left(q_{2}^{\star}, t_{2}\right)-\left[v\left(q_{1}^{\star}, t_{2}\right)-v\left(q_{1}^{\star}, t_{1}\right)\right]=p_{1}^{\star}+\left[v\left(q_{2}^{\star}, t_{2}\right)-\right.$ $\left.v\left(q_{1}^{*}, t_{2}\right)\right]$.

Figure 1, which is adapted from Maskin and Riley (1984), demonstrates graphically the solution for the one-dimensional case with $N=2$. At this point, we focus only on the solid curves in that figure. The monopolist would like to extract all consumer surplus by offering product qualities $q_{1}^{* *}$ and $q_{2}^{* *}$ and charging prices $p_{1}^{* *}$ and $p_{2}^{* *}$, but with such an offering the high type would prefer to mimic the low and select $q_{1}^{* \star}$ (note for a given quality, consumer utility is higher the lower on the figure they can locate). The constrained optimum is given by variables with single asterisks. As above, the high type continues to consume the efficient quality (and pays a lower price), but quality to the low type is degraded, from $q_{1}^{\star \star}$ to $q_{1}^{*}$.

\footnotetext{
${ }^{5}$ We make the usual curvature assumptions $v_{1}>0, v_{11} \leq 0, v_{2}>0, c^{d}>0$, and $d^{\prime \prime}>0$, as well as the normalization that $v\left(0, t_{i}\right)=0$ for all $i$. Furthermore, we maintain the standard single-crossing condition that $u_{q t}>0$, which implies that higher types have greater willingness to pay (WTP) for quality at any price or that consumers may be ordered by their type, $t$.
} 


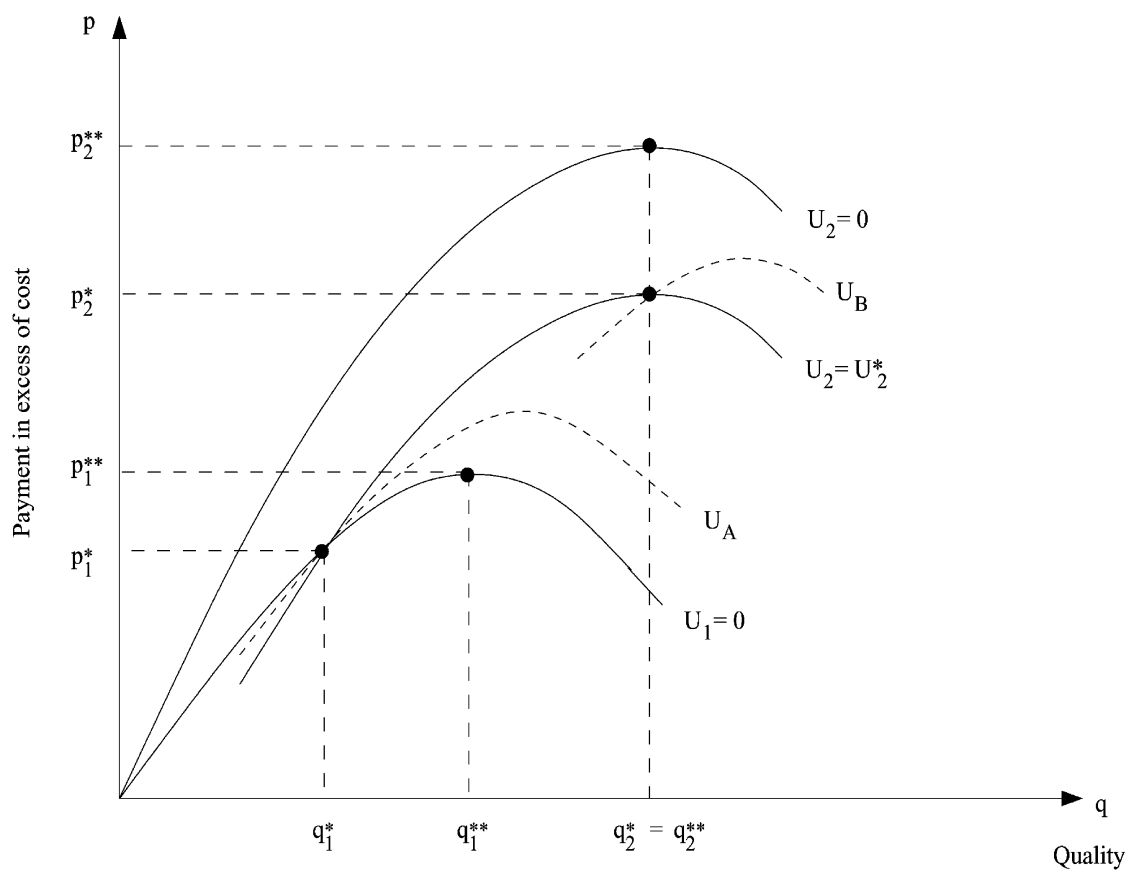

Figure 1. Quality degradation with two types adapted from Maskin and Riley (1984)

\subsection{Continuous Types but Discrete Qualities}

The theory described in the previous section applies also to the case of continuous types but to discrete qualities. To see this, suppose instead that consumer types are continuously distributed on $[\underline{T}, \bar{T}]$ with probability density function $f(t)$ but that the monopolist has decided to offer just two qualities regardless. He or she may do so for a number of reasons. There may be fixed costs associated with the design, production, or marketing of products of different qualities. Or there may be incremental (especially marketing) costs of offering numerous goods. If these are large, the monopolist will offer only those products that can cover his or her fixed costs, limiting the number of products in the market (Spence 1980; Dixit and Stiglitz 1977).

Suppose the firm offered arbitrary qualities $\bar{q}_{1}$ and $\bar{q}_{2}$. Who would buy these goods? All consumers for whom $u\left(\bar{q}_{2}, t\right) \geq u\left(\bar{q}_{1}, t\right)$ and $u\left(\bar{q}_{2}, t\right) \geq 0$ would buy good 2. Because of the structure of the problem-notably the single-crossing condition-only the first of these constraints would bind. Let $\bar{t}_{2}$ denote the consumer type that is just indifferent between purchasing the two goods and $\bar{t}_{1}$ denote the analogous consumer type just indifferent between purchasing good 1 and the outside (or no) good. Then the share of the distribution of consumer types that purchase each good, $\bar{f}_{i}$, is given by the integral under the distribution 


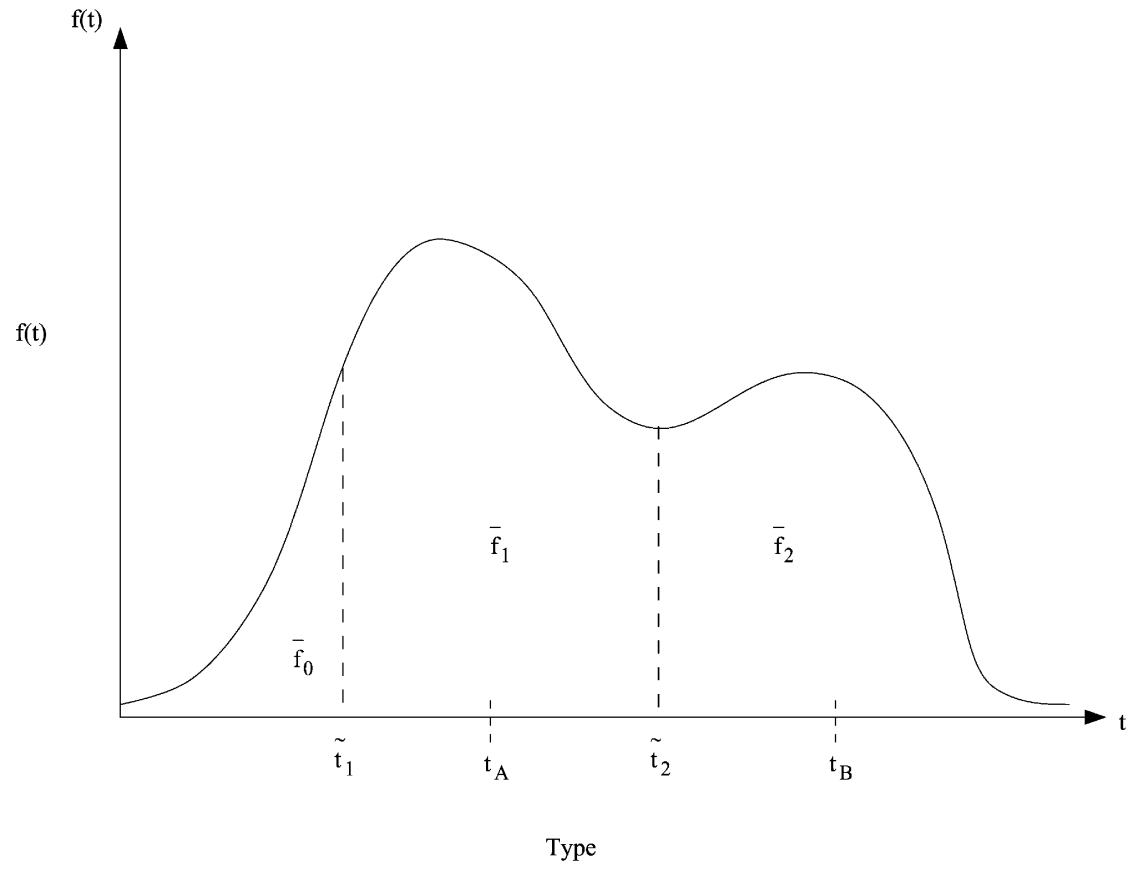

Figure 2. Continuous types and discrete qualities

between the type cut points: $\bar{f}_{i}=\int_{\bar{t}_{i+1}} f(t) d t$ (defining $\bar{t}_{0}=\underline{T}$ and $\bar{t}_{3}=\bar{T}$ ). Figure 2 presents a graphical representation of this framework. In that figure, type $t_{\mathrm{A}}$ lies between the cut types $\bar{t}_{1}$ and $\bar{t}_{2}$ and so consumes the lower bundle. Type $t_{\mathrm{B}}$ lies above the larger cut type $\bar{t}_{2}$, and like that type consumes the higher bundle. For both types $t_{\mathrm{A}}$ and $t_{B}$ (and for all types other than the cut types $\bar{t}_{1}$ and $\bar{t}_{2}$ ), both the participation and incentive constraints hold strictly. The key result is that given these qualities $\bar{q}_{1}$ and $\bar{q}_{2}$ and associated shares $\bar{f}_{0}, \bar{f}_{1}$, and $\bar{f}_{2}$, the monopolist's profit is described by equation (5) just as in the discrete-type case. ${ }^{6}$

An important consequence of continuous consumer types is that quality distortion will generally occur for almost all consumers. In particular, only the highest cut type $\bar{t}_{2}$ will consume an efficient quality $\left(\bar{q}_{2}^{\star}=\bar{q}_{2}^{\star \star}\right)$. All other types $t>\bar{t}_{2}$ that also purchase the high-quality good (like $t_{\mathrm{B}}$ ) will necessarily receive inefficiently low qualities. Similarly, while quality will still be degraded to the lower cut type $\left(\bar{q}_{1}^{\star}<\bar{q}_{1}^{\star *}\right)$, it will be lower still for other, higher, types (like $t_{\mathrm{A}}$ ) that also purchase the low-quality good, $\bar{t}_{1}<t<\bar{t}_{2}$. This is also illustrated in

${ }^{6}$ This is a subtle point. Were we to specify a particular continuous distribution of consumer types, solving the firm's problem for the optimal cut types, $\bar{t}$ 's, is a challenging problem requiring more sophisticated techniques than those employed here (Crawford and Shum 2005). The insight is that even if firms are making these more sophisticated calculations, the discrete-type first-order conditions must hold for the cut types ultimately chosen by firms. 
Figure 1, in which the two dashed curves are indifference curves for the types $t_{\mathrm{A}}$ and $t_{\mathrm{B}}$ in Figure 2. Type $t_{\mathrm{A}}$, who consumes the same bundle as type $\bar{t}_{1}$, has an efficient bundle that lies to the right of type $\bar{t}_{1}$ 's efficient bundle, which implies that the quality distortion to type $t_{\mathrm{A}}$ is higher than that to type $\bar{t}_{1}$. Similarly, there is a positive distortion to type $t_{\mathrm{B}}$, even though he or she consumes the same bundle as type $\bar{t}_{2}$, to whom there is no distortion.

The theory described above applies analogously for an arbitrary number $n$ of offered qualities. For any $n$, equation (6) continues to hold, with associated degradation for all but the highest offered quality $q_{n}$. However, when the type distribution is continuous but the monopolist offers only discrete qualities, the cut types $\bar{t}_{1}$ and $\bar{t}_{2}$, as well as $n$, the number of offered qualities, are also choice variables. In this paper, while we do not use the monopolist's optimality conditions for these variables in recovering quality measures, we do briefly analyze the number of goods offered by firms in the empirical analysis.

Finally, note that it is typical in models of this type to make additional assumptions on the distribution of consumer types to ensure the optimal prices and qualities are monotonically increasing in types. Because, however, we restrict our attention to the implications of the model for a discrete number of qualities, we do not have to do this. Indeed, it could be the case that in some market $n$ the inverse hazard function of types, $\left[1-F_{n}(t)\right] / f_{n}(t)$, is nonmonotonic in $t$ (as in Figure 2). If the firm in market $n$ were to offer a fully nonlinear price/quality schedule in such a case, it would require sophisticated solution techniques involving pooling of types at particular qualities (Wilson 1993). With discrete qualities, however, pooling obtains regardless of the shape of the type distribution. For our purposes, it is convenient if the inverse hazard function defined by the share of households between cut types in each market, the $\bar{f}$, is monotonic. This is true for the majority of the markets in our data. ${ }^{7}$

\subsection{Quality Choice and Regulation}

In a pair of papers, Besanko, Donnenfeld, and White $(1987,1988)$ extend the Mussa-Rosen model to consider the monopolist's quality choice problem in the presence of regulation. They consider three forms of regulation-minimum quality standards (MQSs), maximum price (price cap) regulation, and rate-of-return regulation-the first of which is most likely to apply to the cable television industry in the period we analyze. ${ }^{8}$ We briefly describe here the consequences of MQSs for firm behavior and market outcomes in the context of the MussaRosen model with discrete goods.

Suppose regulation forbids the sale of goods with quality below a given level q. This introduces a set of constraints, $q_{i} \geq q, \forall i$, on the monopolist's objective

\footnotetext{
${ }^{7}$ If it does not hold, the optimal solution may have pooling of the cut types at a common quality. We verify this is not the case for each market in the sample.

${ }^{8}$ Section 3 discusses in more detail cable regulations in force during the period of this study.
} 
function given in equation (5). Under our assumptions, these constraints may bind only for the lowest quality good offered to consumers.

Let $\lambda$ be the Lagrange multiplier associated with the minimum quality constraint, $q_{1} \geq q$, for the lowest offered quality, $q_{1}$. Then the monopolist's firstorder conditions for qualities (and associated prices) are as above except for $q_{1}$, which is now

$$
S_{q}\left(q_{1}, t_{1}\right)+\lambda=\frac{1-F_{1}}{f_{1}}\left[v_{q}\left(q_{1}, t_{2}\right)-v_{q}\left(q_{1}, t_{1}\right)\right] .
$$

At the optimum, $\lambda$ is set to ensure that $q_{1}$ is at least $q$ (and is zero if the unconstrained $\left.q_{1}>q_{1}\right)$. The remaining qualities and prices follow from the unconstrained optimum (with $q_{1}=q$ ). As we shall see, this is convenient for empirical measurement of the consequences of regulation on quality choice.

\section{The Cable Television Industry}

Cable television systems bundle television networks into services and offer these services to households in local (geographically separate) cable markets. ${ }^{9}$ The largest and most popular services are called basic and expanded basic services (or tiers) and contain broadcast and (so-called) cable television networks. ${ }^{10}$ Consumers can also purchase premium services, which are advertising-free entertainment networks typically offering full-length feature films and original programming and offered on a stand-alone basis (examples include $\mathrm{HBO}$ and Showtime). To buy any cable services, all households must first purchase basic service. In practice, the majority also purchase one or more expanded basic and/ or premium services.

There are two dimensions to systems' choice of service quality: the quality of the program networks being offered and the allocation of those networks into service bundles. This paper focuses on the quality of a given set of program bundles. ${ }^{11}$ The institutional and economic environment in the industry suggests that the design of basic and expanded basic services maps well to the theory

\footnotetext{
${ }^{9}$ Most cable television systems now compete with two major direct-broadcast satellite services in the multichannel video-programming distribution (MVPD) market. Satellite services, however, only became viable competitors to cable systems with the passage of the Satellite Home Viewer Improvement Act in 1999, which permitted them to distribute local broadcast television signals within local television markets. The data used in this paper are from 1995, a time when satellite providers had an aggregate 3.4 percent share of the MVPD market (FCC 2001), with these often drawn from rural markets where there was no offered cable service. In addition, approximately 1-2 percent of cable markets have a second wireline cable provider (FCC 2005b, n. 627). None of the systems we consider are from these so-called overbuilt markets.

${ }^{10}$ Broadcast networks are television signals broadcast in the local cable market and then collected and retransmitted by cable systems. Examples include the major national broadcast networks-ABC, CBS, NBC, and FOX - as well as public and independent television stations. Cable networks are advertising-supported general and special-interest networks that are distributed nationally to systems via satellite, such as MTV, CNN, and ESPN.

${ }^{11}$ In related work, Crawford (2004) analyzes the bundling decision in the cable television industry.
} 
described in Section 2. Since households that buy expanded basic services must necessarily first purchase basic service, these services are by construction increasing in overall quality. As such, consumer preferences may be adequately described by a single vertical dimension measuring overall tastes for multichannel television. Furthermore, since they consist of generally large bundles of individual networks, the range of qualities possibly chosen is plausibly continuous, but the offered qualities are clearly discrete. Finally, they are the largest and most important source of revenue for cable systems (Kagan World Media 2002).

While basic and expanded basic services map well to the theory, systems also offer premium services. These are not bundled, however, and households can select among them as long as they purchase at least basic service. As such, they may be considered a horizontally differentiated set of alternatives to the vertical sequence of basic and expanded basic services. Unfortunately, solving for the optimal prices and qualities for products with multiple dimensions of differentiation requires models of multidimensional screening that have proven to be extremely challenging (Rochet and Stole 2000; Rochet and Choné 1998). ${ }^{12}$ In the balance of the paper, we therefore focus on measuring quality degradation for just basic and expanded basic cable television services and note that our results about quality degradation are conditional on considering the truncated menu of cable services.

Consumer dissatisfaction with various aspects of cable television services is common. Fuelling demands for regulatory oversight is the persistent growth in cable prices over time. Between 1986 and 1991, 1992 and 1996, and 1996 and 2002 , respectively, cable prices rose 11.6, 2.2, and 7.1 percent annually, far faster than rates of price inflation for comparable consumer nondurables in the same period (GAO 1991; FCC 1997, 2003).$^{13}$ Of course, the quality of cable services has also increased. While difficult to quantify directly, the number of cable networks, expenditure on cable programming, and viewership of cable networks have all grown substantially over this period (Hazlett and Spitzer 1997). That being said, the independent American Consumer Satisfaction Index (ACSI) concludes that cable "is one of the worst-performing industries in the ACSI" and that "people are very dissatisfied" (Consumer Reports 2002, p. 33). Consumers regularly complain that price increases outstrip quality increases, particularly for new channels they feel they will not watch (Horn 2001). Many appear to want "fewer channels [and] lower rates" (Fowler 2000, p. AC5). We present preliminary evidence of quality degradation from the raw data in the next section.

Reflecting these frustrations, cable television service has periodically been sub-

\footnotetext{
${ }^{12}$ Assessing the impact of ignoring premium services is itself a challenge. Rochet and Stole (2002) suggest that providing households with outside options moderates the monopolist's incentives to degrade quality. Premium services, however, are outside options under the monopolist's control, which presumably moderates this effect.

${ }^{13}$ This is despite price regulations mandating 17 percent price reductions by the 1992 Cable Television Consumer Protection and Competition Act of 1992 (47 U.S.C. sec. 533) for the middle period.
} 
ject to regulatory oversight. In 1995, the year of our data, two dimensions of cable service were subject to regulations promulgated by the Cable Television Consumer Protection and Competition Act of 1992 (47 U.S.C. sec. 533). The first required systems to offer a service tier containing at minimum all available broadcast stations in their market. ${ }^{14}$ For systems offering a single (basic) service, this requirement was not binding, as that service already contained these plus (sometimes many) additional networks. For some systems offering expanded basic services, however, this requirement served as an MQS. Many systems responded by introducing new limited basic or lifeline basic services to satisfy the rule. Beyond this restriction, systems may select and package whatever television networks they like for sale to households.

The 1992 cable act also introduced price regulations for basic and expanded basic services. These mandated that cable systems reduce their per-channel basic and expanded basic prices by 17 percent from September 1992 levels but only if the local franchise authority certified to regulate rates with the FCC (for basic services) or consumers submitted complaints to the FCC (for expanded basic services). ${ }^{15}$ Because of a combination of factors, including strategic responses by cable systems, relatively weak cost pass-through (going-forward) requirements, and generous "social contracts" between regulators and operators, price regulation provided little price relief to households. ${ }^{16}$ The Telecommunications Act of 1996 (Pub. L. No. 104-104, 110 Stat. 56 [1996]) subsequently removed even these price controls on systems. While systems now face little direct regulatory scrutiny, there remains a constant threat of reregulation, which may itself influence system behavior (McConnell 2003). In this paper, certification by a local franchise authority to regulate rates in the early 1990s likely measures not only the goal of regulating low-quality services but also the presence of active local concern about cable prices and quality.

\subsection{Data}

We have compiled a market-level data set on a cross section of U.S. cable systems to evaluate quality degradation in the industry. The primary source of data for these systems is Warren Publishing's Television and Cable Factbook. The data for this paper consist of the population of cable systems recorded in Warren

\footnotetext{
${ }^{14}$ Among these are included stations that demanded carriage (so-called must-carry stations) as well as those that negotiated rights for carriage (retransmission consent stations). The former tended to be smaller independent stations unaffiliated with one of the four major broadcast networks.

${ }^{15}$ The 1992 cable act introduced a split regulatory structure, with local franchise authorities given authority to regulate rates of basic service and federal regulators given authority to regulate rates of expanded basic services. Franchise authorities wanting to regulate basic service rates were required to certify with the FCC. See Johnson (1994) and Crandall and Furchtgott-Roth (1996) for more details about the 1992 cable act.

${ }^{16}$ See Hazlett and Spitzer (1997) and Crawford (2000) for more details on the consequences to cable television prices and qualities of the these regulations.
} 
(1996) for which complete information was available. ${ }^{17}$ A sample of 1,042 systems remained. ${ }^{18}$

Table 1 presents sample statistics for selected variables from these systems. Systems in the sample offer at most three basic and expanded basic services. While all systems offer basic service, 30 percent offer at least one expanded basic, and 7 percent offer two. ${ }^{19}$

Cable services contain an increasing set of stations, with the smallest bundles offering a subset of the stations offered in the larger bundles. For example, for markets offering three goods, we define a system's high-quality product to contain all three basic and expanded basic services, with associated prices and market shares indexed by the number 3 . We similarly define a system's medium-quality products, indexed by 2 , to contain the first two offered services, and its lowquality products, indexed by 1 , to contain just the first offered service. Products are defined analogously (that is, from the top) in two- and one-good markets. In what follows, we order the quality levels in increasing order: $q_{4-n}<\cdots<q_{3}{ }^{20}$ As can be seen across columns in Table 1, most households purchase all offered expanded basic services, although there are significant sales of lower quality services in three-good markets.

Table 1 presents the average number of cable, broadcast, and other channels offered on each available basic service. ${ }^{21}$ Aggregating over all basic and expanded basic services, systems typically offer almost six broadcast networks, more than 17 cable networks, and almost 14 other networks. The top 15 cable programming networks available in the United States in 1998 are listed in Table 2.

\subsection{Measuring Cable Service Quality}

Measuring service quality is notoriously difficult in the cable television industry. Much of the recent empirical literature measures quality by the number

\footnotetext{
${ }^{17}$ While there are over 11,000 systems in the sample, persistence in nonresponse over time as well as incomplete reporting of critical variables required imposing a large number of conditions in order for a system to be included in each sample. Missing information on prices, quantities, and reporting dates were responsible for the majority of the exclusions. See Crawford (2000) for more information about the sampling procedure.

${ }^{18}$ We also excluded 122 observations for which our procedure recovered qualities that were nonmonotonic in types. This was largely driven by very small market shares (some less than .1 percent) for low-quality goods, which violated the assumption of an increasing hazard function required by the theory. It is likely that such services represented a compromise between systems and regulators seeking services affordable to low-income households. As this is outside the scope of our model, we dropped these systems from the analysis.

${ }^{19}$ Designations for expanded services have little meaning. The data report expanded services in the order input by systems. In practice, Expanded Basic I tends to have more programming, a higher price, and a higher market share than Expanded Basic II.

${ }^{20}$ Because the theory implies no distortion at the top, it is convenient to adopt this indexing convention over the alternative $q_{1}<\cdots<q_{n}$.

${ }^{21}$ Note that reported cable networks is the number of networks among the top 40 most popular as of 1998 (top-40 networks), reported broadcast networks are offered only on the lowest quality (basic) service, and other networks are public, educational, and government channels and other cable networks outside the top 40 .
} 
Table 1

Sample Statistics: Selected Characteristics

\begin{tabular}{|c|c|c|c|c|}
\hline Variable & $\begin{array}{c}\text { All } \\
\text { Markets }\end{array}$ & $\begin{array}{l}\text { Three-Good } \\
\text { Markets }\end{array}$ & $\begin{array}{l}\text { Two-Good } \\
\text { Markets }\end{array}$ & $\begin{array}{c}\text { One-Good } \\
\text { Markets }\end{array}$ \\
\hline \multicolumn{5}{|l|}{ Expanded basic services: } \\
\hline Any & .30 & 1.00 & 1.00 & .00 \\
\hline One & .23 & .00 & 1.00 & .00 \\
\hline Two & .07 & 1.00 & .00 & .00 \\
\hline \multicolumn{5}{|l|}{ Market shares: } \\
\hline$w_{3}$ & .66 & .47 & .61 & .70 \\
\hline$w_{2}$ & $.06^{\mathrm{a}}$ & .12 & .04 & $\ldots$ \\
\hline$w_{1}$ & $.04^{\mathrm{a}}$ & .04 & $\ldots$ & $\ldots$ \\
\hline \multicolumn{5}{|l|}{ Prices: } \\
\hline$p_{3}$ & 20.40 & 25.64 & 22.69 & 19.13 \\
\hline$p_{2}$ & $14.32^{\mathrm{a}}$ & 21.86 & 12.05 & $\ldots$ \\
\hline$p_{1}$ & $16.78^{\mathrm{a}}$ & 16.78 & $\ldots$ & $\ldots$ \\
\hline \multicolumn{5}{|l|}{ Programming: } \\
\hline \multicolumn{5}{|l|}{ Top 40 cable networks: } \\
\hline On service 3 & 16.55 & 22.29 & 20.85 & 14.57 \\
\hline On service 2 & $9.14^{\mathrm{a}}$ & 18.15 & 6.44 & $\ldots$ \\
\hline On service 1 & $11.94^{\mathrm{a}}$ & 11.94 & $\ldots$ & $\ldots$ \\
\hline \multicolumn{5}{|l|}{ Broadcast networks: } \\
\hline Over the air & 2.54 & 3.19 & 2.85 & 2.37 \\
\hline On cable & 5.74 & 6.57 & 6.51 & 5.40 \\
\hline Other networks on basic & 14.36 & 10.85 & 13.18 & 15.09 \\
\hline \multicolumn{5}{|l|}{ System characteristics: } \\
\hline Homes passed $(1,000 \mathrm{~s})$ & 5.11 & 9.54 & 12.19 & 2.34 \\
\hline Channel capacity & 38.87 & 43.21 & 43.55 & 36.91 \\
\hline \multicolumn{5}{|l|}{ Market characteristics: } \\
\hline \multicolumn{5}{|l|}{ Income: } \\
\hline Mean & 28.83 & 28.27 & 30.11 & 30.15 \\
\hline Standard deviation & 24.61 & 24.15 & 25.52 & 26.21 \\
\hline Skew & 2.69 & 2.70 & 2.64 & 2.68 \\
\hline \multicolumn{5}{|l|}{ Age: } \\
\hline Mean & 36.22 & 36.36 & 35.75 & 36.34 \\
\hline Standard deviation & 23.20 & 23.36 & 22.74 & 23.03 \\
\hline Skew & .16 & .15 & .18 & .15 \\
\hline \multicolumn{5}{|l|}{ Household size: } \\
\hline Mean & 2.67 & 2.66 & 2.67 & 2.65 \\
\hline Standard deviation & 1.60 & 1.61 & 1.59 & 1.61 \\
\hline Skew & 1.78 & 1.76 & 1.81 & 1.92 \\
\hline Rural share & .63 & .66 & .56 & .55 \\
\hline Certification & .12 & .36 & .15 & .08 \\
\hline$N$ & 1,042 & 72 & 240 & 730 \\
\hline
\end{tabular}

Note. Data on cable systems, including service, market share, price, and programming data, are from Warren Publishing (1996). Prices are in 1995 dollars. Data on demographic information are from the U.S. Census Bureau (1994). Certification is a dummy variable that indicates the local franchise authority registered with the Federal Communications Commission (FCC) to regulate basic cable rates as of April 1996. Data on certification are from the Cable Services Bureau, FCC, April 1996.

${ }^{a}$ Averaged over markets offering this service. 
Table 2

Top 15 Cable Programming Networks

\begin{tabular}{llll}
\hline Rank & \multicolumn{1}{c}{ Network } & Subscribers (millions) & Programming Format \\
\hline 1 & TBS Superstation & 77.0 & General interest \\
2 & Discovery Channel & 76.4 & Nature \\
3 & ESPN & 76.2 & Sports \\
4 & USA Network & 75.8 & General interest \\
5 & C-SPAN & 75.7 & Public affairs \\
6 & TNT & 75.6 & General interest \\
7 & FOX Family Channel & 74.0 & General interest/kids \\
8 & TNN (The Nashville Network) & 74.0 & General interest/country \\
9 & Lifetime Television & 73.4 & Women's \\
10 & CNN (Cable News Network) & 73.0 & News \\
11 & A\&E & 73.0 & General interest \\
12 & The Weather Channel & 72.0 & Weather \\
13 & QVC & 70.1 & Home shopping \\
14 & The Learning Channel (TLC) & 70.0 & Science \\
15 & MTV: Music Television & 69.4 & Music \\
\hline
\end{tabular}

Note. Data on network subscribers are from National Cable and Telecommunications Association, Top 20 Cable Programming Networks - as of December 2006 (http://www.ncta.com/ContentView.aspx ?contentID =74). Data on programming formats from individual network promotional material are from National Cable and Telecommunications Association, Cable Networks (http://www.ncta.com/Organizations.aspx?type $=$ orgtyp2\&contentID $=2907$ ) or industry sources.

of channels offered on each service (Mayo and Otsuka 1991; Rubinovitz 1993; Hazlett and Spitzer 1997). While this can be dangerous-it assumes all cable networks are of equal underlying quality-it is a useful summary measure that we can use to look for evidence of quality degradation in our raw data.

Table 3 reports the average price paid per channel for each offered cable service in three- and two-good cable markets. ${ }^{22}$ Channels included in the calculations are all the major broadcast networks and the top 40 satellite networks as of 1998. Under the assumption that each channel is of the same underlying quality, quality degradation would be reflected in higher per-channel prices for low- and medium-quality services.

The results suggest moderate quality distortion in low- and medium-quality cable services. Table 3 reports results using the total of prices and channels for each offered services. In three-good markets, on average across markets, the price per channel rises modestly from the highest to lowest quality service. Despite these seemingly small differences, we can reject the null hypothesis of equal prices per channel across services at conventional significance levels. In twogood markets, the results are even stronger. The price per channel of the highquality service is less than two-thirds of the low-quality service, a very large (and statistically significant) difference.

While these results are suggestive, elsewhere we find that the identities of offered channels are very important in describing cable service quality and de-

\footnotetext{
${ }^{22}$ Recall the Mussa-Rosen model predicts no quality degradation in one-good markets. For reference, the average price per channel in these markets is $\$ 1.38$.
} 
Table 3

Preliminary Evidence of Quality Degradation

\begin{tabular}{|c|c|c|c|c|}
\hline \multirow[b]{2}{*}{ Total Prices/Channels } & \multicolumn{2}{|c|}{$\begin{array}{c}\text { Three-Good } \\
\text { Markets }\end{array}$} & \multicolumn{2}{|c|}{$\begin{array}{l}\text { Two-Good } \\
\text { Markets }\end{array}$} \\
\hline & Mean & Difference & Mean & Difference \\
\hline$p_{3} /$ channel & $1.06(.03)$ & $-.04(.01)$ & $1.10(.03)$ & $-.61(.06)$ \\
\hline$p_{2} /$ channel & $1.10(.03)$ & $-.13(.02)$ & $1.72(.07)$ & $\ldots$ \\
\hline$p_{1} /$ channel & $1.23(.04)$ & & . . & \\
\hline$N$ & 72 & & 239 & \\
\hline
\end{tabular}

Note. Reported are the average price per channel for each offered cable service. Channels include all top 40 satellite channels and, for the lowest quality service, all major broadcast networks. Ratios are formed with total price and total channels. Values in the Difference columns are the difference in price per channel in that row and the row that follows. The cable system in one two-good market included no satellite or broadcast networks in its lowest quality service. Standard errors are in parentheses.

mand (Crawford 2000). ${ }^{23}$ As such, in this paper, we directly measure cable service quality by exploiting the restrictions of a theoretical model of optimal price and quality choice to recover quality measures consistent with the observed prices and market shares. We then relate these to the networks available to determine the incremental quality offered by each. In addition to controlling for heterogeneity in network quality, we can exploit this model to directly measure quality degradation by systems. These procedures are described in further detail in the following sections.

\section{Empirical Model and Algorithm for Measuring Quality}

\subsection{Empirical Model}

Following Mussa and Rosen (1978), we assume that utility for a consumer of type $t$ is linear in quality,

$$
u\left(q_{i}, t\right)=t q_{i}-p,
$$

and costs to the firm are quadratic in quality,

$$
C\left(q_{i}\right)=\frac{1}{2} q_{i}^{2}
$$

Given these assumptions, total surplus from a consumer of type $t$ is $S\left(q_{i}\right.$, $t) \equiv t q_{i}-.5 q_{i}^{2}$. In this specification, $t$ measures consumer willingness to pay (WTP) for quality net of firm's marginal cost for quality. ${ }^{24}$ For convenience, we

\footnotetext{
${ }^{23}$ For example, we find mean WTP for popular channels like ESPN and Nickelodeon up to 10 times as great as that for channels like the Discovery Channel and MTV.

${ }^{24}$ In other words, for this specification, preference levels cannot be separately identified from marginal cost levels. This is a typical problem in the empirical analysis of product markets (Bresnahan 1989). This is not a great concern in this paper, as we focus on observable outcomes (prices, qualities, and market shares) that are invariant to the composition of $t$. It would matter, however, in calculating the profit and welfare consequences of observable price/quality schedules and/or associated regulatory interventions.
} 
refer to the type parameter, $t$, as net WTP. This specification is also convenient because the socially optimal quality for any type $t$ is given by $S_{q}\left(q_{i}, t\right)=0$, which implies $q_{i}^{* *}=t$. Therefore, given measures of cut types $\bar{t}_{i}$ and qualities $q_{i}$, one can immediately measure the extent of quality distortion (for the cut type) as the difference $\left(\bar{t}_{i}-q_{i}\right)$.

The Mussa-Rosen assumptions are convenient as they yield closed-form solutions for optimal qualities and prices as a function of these net types. They are also fairly restrictive, however, particularly in the assumption of quadratic costs of providing quality. We justify them on two grounds. First, they accurately reflect the nature of costs in the industry. The primary marginal quantity (unit) cost to cable systems are the per-subscriber monthly affiliate fees paid to networks for carrying their programming. Offering a service that is higher in quality means offering more and/or better cable networks, at increasing unit cost. Furthermore, the most valuable (highest quality) networks tend to have more bargaining power with cable systems, which suggests that costs are convex in quality. ${ }^{25}$ Regardless, as the curvature of the cost function is important for determining quality degradation in the Mussa-Rosen model, in the Appendix we evaluate the sensitivity of our conclusions to our assumptions on its shape.

The second justification for our assumptions is that they allow us considerable flexibility in estimating the distribution of net preferences across markets. In particular, we treat each cable market in isolation and recover the distribution of types in that market consistent with observed market shares and prices. Given that we observe only a fixed number of bundles $n$ being offered in any given market, it is clear that we would not be able to identify (nonparametrically) any continuous-type distribution completely but rather only the indifferent consumers $\bar{t}_{4-n}, \ldots, \bar{t}_{3}$ and the corresponding percentiles $\bar{f}_{4-n}, \ldots, \bar{f}_{3}{ }^{26}$ Such flexibility is important, however, as it is variation in the distribution of tastes across markets that drives differences in offered qualities. ${ }^{27}$

\subsection{Recovering Implied Qualities}

Our basic strategy is to recover values for the net type distribution from observed prices and market shares in each market. The estimating equations are derived from the Mussa-Rosen model of optimal quality choice. As such, once

\footnotetext{
${ }^{25}$ Indeed, a graph of affiliate fees for the 40 top cable networks in 1995 is clearly convex (Kagan World Media 2004).

${ }^{26}$ For these reasons, we do not exploit the model to predict the optimal number of services to offer. To do so would require simulating the profit associated with other offered qualities. This in turn requires information about the type distribution between the recovered cut types. We do, however, ensure that for the type that we recover, it would not be more profitable for the monopolist to offer fewer services (by pooling over types).

${ }^{27}$ In ongoing work (Crawford and Shum 2005) using more recent data, we allow preferences to be continuously distributed within markets and pool information across markets to flexibly estimate features of those distributions. Preliminary results from the model in that paper applied to the data in this paper yield results qualitatively similar to those presented here.
} 
we have the type distribution, it is a simple matter to calculate the offered qualities implied by the model.

As described earlier, we recover parameters separately for each market. In each market $c$, the unknown parameters are the cut types in the distribution of net consumer preferences, $\bar{t}_{i c}$ and the associated mass between these types, $\bar{f}_{i c}$, for $i=4-n, \ldots, 3$ and $c=1, \ldots, C$. Let $\theta_{c} \equiv\left\{\bar{f}_{i c}, \bar{t}_{i c} ; i=4-\right.$ $\left.n_{c}, \ldots, 3\right\}$ describe the vector of parameters in market $c$. There are $2 n_{c}$ parameters per market, where $n_{c}$ is the number of services offered by the system in market $c$. Importantly, we allow the parameters $\theta_{c}$ to vary across markets $c$, which allows the distribution of consumer types $t$ to differ across cities.

The available data in each market consist of market shares and prices of the $n_{c}$ bundles $\left\{s_{i c}, p_{i c} ; i=4-n, \ldots, 3\right\}$. In three-good markets, we have six parameters and six observed variables; in two-good markets, we have four parameters and four variables; and so on. Thus the economic model is just identified in each market. For convenience, in the description to follow, we omit the market subscript $c$.

For each market, the parameters $\bar{f}_{i} ; i=4-n, \ldots, 3$ can be immediately recovered from the observed market shares as

$$
s_{i}=\bar{f}_{i}, \quad i=4-n, \ldots, 3,
$$

so that the market share of the outside good is given by $s_{0}=1-\sum_{i=4-n}^{3} \bar{f}_{i}$. In order to solve for the $n$ cut types $\bar{t}_{i}, i=4-n$, 3, we note that the prices are characterized as

$$
p_{i}=v\left(q_{i}, \bar{t}_{i}\right)-u_{i}\left(q_{i}\right)=\bar{t}_{i} q_{i}-u_{i}\left(q_{i}\right)
$$

where $q_{i}$ is endogenously determined by the firm. Given knowledge of $\bar{f}_{i}$ using equation (10) above, the (unknown) quality levels $q_{i}$ are given by the first-order conditions given in equation (6):

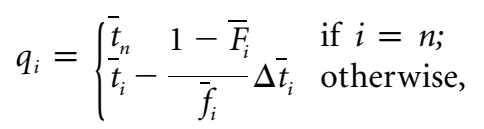

where $\bar{F}_{i}=\sum_{i \equiv 0}^{i} \bar{f}_{i}$ and $\Delta \bar{t}_{i} \equiv \bar{t}_{i+1}-\bar{t}_{i^{\prime}}$. Moreover, the associated utility levels for the cut types, $\bar{u}_{i}, i=4-n, \ldots, 3$, are

$$
\begin{aligned}
\bar{u}_{i} \equiv u\left(q_{i}, w \bar{t}_{i}\right) & =u\left(q_{i-1}, \bar{t}_{i}\right), \\
& =u\left(q_{i-1}, \bar{t}_{i-1}\right)+u\left(q_{i-1}, \bar{t}_{i}\right)-u\left(q_{i-1}, \bar{t}_{i-1}\right), \\
& =u\left(q_{i-1}, \bar{t}_{i-1}\right)+\Delta \bar{t}_{i-1} q_{i-1}=\ldots= \\
& =u\left(q_{1}, \bar{t}_{1}\right)+\sum_{i^{\prime}=1}^{i-1} \Delta \bar{t}_{i^{\prime}} q_{i^{\prime}}=\sum_{i^{\prime}=1}^{i-1} \Delta \bar{t}_{i^{\prime}} q_{i^{\prime}},
\end{aligned}
$$

where the final equality obtains from the indifferent condition for cut type $\bar{t}_{1}$, which is $u\left(q_{1}, \bar{t}_{1}\right)=0$. 
By plugging equation (12) into equation (13), and then substituting the implied forms of $q_{i}$ and $u_{i}$ into the price equations (11), we obtain a system of $n$ nonlinear equations that can be solved for the $n$ unknowns $\bar{t}_{i}, i=4-n, 3$. Note that since our values of $\bar{f}_{i}, \bar{t}_{i}, i=4-n, 3$ are just transformations of the observed data (albeit very nonlinear ones in the case of the $\bar{t}_{i}^{\prime} \mathrm{s}$ ), there are no standard errors for these measures. ${ }^{28}$

The intuition for how we recover implied qualities from the data may be described by a simple example. Consider a market with two offered qualities. In this market, we observe three market shares (including that of the outside good), $s_{0}, s_{1}$, and $s_{2}$, and two prices, $p_{1}$ and $p_{2}$. From these, we wish to recover information about the net type distribution, a discrete distribution with three points of support characterized by five parameters, $\bar{f}_{0}, \bar{f}_{1}, \bar{f}_{2}, \bar{t}_{1}$, and $\bar{t}_{2}{ }^{29}$ Our procedure asks, What five values for the net type distribution in this market can rationalize the five observed data points under the Mussa-Rosen model? Once we have answered that question and obtained the net type distribution, the theory permits us to calculate the implied qualities for each product in the market. We then repeat this process for each market.

From equation (10), the share of people purchasing each product exactly identifies the share of each type within the market, $\bar{f}_{i}=s_{i}$. The challenge is in recovering the cut types $\bar{t}_{1}$ and $\bar{t}_{2}$. These are obtained from observed prices using the quality first-order conditions in equation (12) and pricing equation (11) as follows. For our two-good example, optimal qualities and prices as a function of the net type distribution are given by

$$
\begin{gathered}
q_{2}=\bar{t}_{2}, \quad p_{2}=\bar{t}_{2}^{2}-\Delta \bar{t} q_{1}, \\
q_{1}=\bar{t}_{1}-\frac{1-\bar{F}_{1}}{\bar{f}_{1}} \Delta \bar{t}, \quad p_{1}=\bar{t}_{1} q_{1} .
\end{gathered}
$$

If the cable system was a perfectly discriminating monopolist, there would be no quality distortion, and solving for the cut types under our modeling assumptions would be trivial: $q_{i}=\bar{t}_{i}$ and $p_{i}=\bar{t}_{i} q_{i}=\bar{t}_{i}^{2}$ for each $i \in\{1,2\}$. Each cut type would then be given by the square root of its observed price, $\bar{t}_{i}=$ $\sqrt{p_{i}}$

There is not, unfortunately, an intuitive representation of this solution for the case of quality degradation described by equation (14). Instead, we provide two illustrative examples. Consider first two markets that have similar prices and shares of consumers who buy either service $\left(\bar{f}_{1}+\bar{f}_{2}\right)$ but differ in the relative

\footnotetext{
${ }^{28}$ In this sense, our empirical procedure resembles the first step in Berry's (1994) procedure, in which mean quality levels for each brand are recovered as a function of market shares, and in Miravete and Röller (2003), in which the parameters of optimal nonlinear tariffs are recovered as a function of the slope and curvature of observed tariff functions.

${ }^{29}$ We cannot technically estimate the boundaries of the support of the type distribution, but we do not need these to calculate implied qualities.
} 
weights of $\bar{f}_{1}$ and $\bar{f}_{2}$. Table 4 provides an example from the data of two such markets. In the high $-f_{1}$ market (Kamas), there are more people with tastes for the low-quality good $q_{1}$, which reduces the monopolist's incentive to degrade its quality. In general, this would also induce the monopolist to increase $p_{1}$ and decrease $p_{2}$. Since prices are similar in both markets, however, the higher $q_{1}$ in the high- $\bar{f}_{1}$ market must be rationalized by lower $\bar{t}_{1}$ (from the equation for $p_{1}$ ) and higher $\bar{t}_{2}$ (from the equation for $p_{2}$ ).

Similarly, consider the two markets in Table 4 with similar market shares and low-quality price $p_{1}$ that differ in the high-quality price $p_{2}$. In this example, even if the number of each type is similar, that is, the $f$ s are similar, the higher $p_{2}$ in Belleville suggests a greater WTP for the high-quality good, $\bar{t}_{2}$, among households there. By making high types more profitable to the monopolist, this would have the tendency to increase degradation in $q_{1}$ and lower $p_{1}$. Since $p_{1}$ is similar in each market, however, it must be that $t_{1}$ is also higher in the high- $p_{2}$ market (although $q_{1}$ is indeed lower).

These examples both show that the primary challenge in our procedure is to separate the impact of WTP for quality across markets, $\bar{t}$ 's, from offered qualities that are themselves determined by those preferences, $q(\bar{t})$ 's. The theory provides the solution: cut types in each market are found that can simultaneously satisfy the monopolists' optimal choice of quality as well as yield prices equal to those observed in the data.

This paper differs from the recent empirical literature analyzing quality choice in several respects. First, the majority of the existing literature focuses on prices and not quality. For example, Verboven (2002), Cohen (2001), and Clerides (2002) compare prices (or markups) for various qualities of cars, paper towels, and books, respectively, to see if there is evidence of price discrimination by quality. Similarly, Leslie (2004) simulates alternative pricing policies for different (but fixed) qualities of a Broadway show. Closest in spirit to our paper is the recent work by McManus (forthcoming), which compares estimated marginal benefits and observed marginal costs of quality variants in specialty coffees and finds evidence of degradation for low-quality variants. We differ, however, in that he tests the implications of the Mussa and Rosen (1978) model, whereas we take it as a maintained assumption to analyze its implications for offered qualities. ${ }^{30}$

Our work is much closer in spirit to (and motivated by) the large recent literature on differentiated product demand estimation using discrete choice random coefficient models of consumer choices (Berry 1994; Berry, Levinsohn, and Pakes 1995). In that literature, the distribution of consumers' WTP for (multiple) characteristics is identified by variation in market shares across products and markets. While identified by the demand side alone, some authors also

\footnotetext{
${ }^{30}$ Methodologically, this approach is most closely related to recent literature applying principalagent models of adverse selection to other problems like nonlinear price quantity schedules (Bousquet and Ivaldi 1997; Miravete 2002; Miravete and Roller 2003) and informational asymmetries in the design of regulatory mechanisms (Wolak 1994).
} 


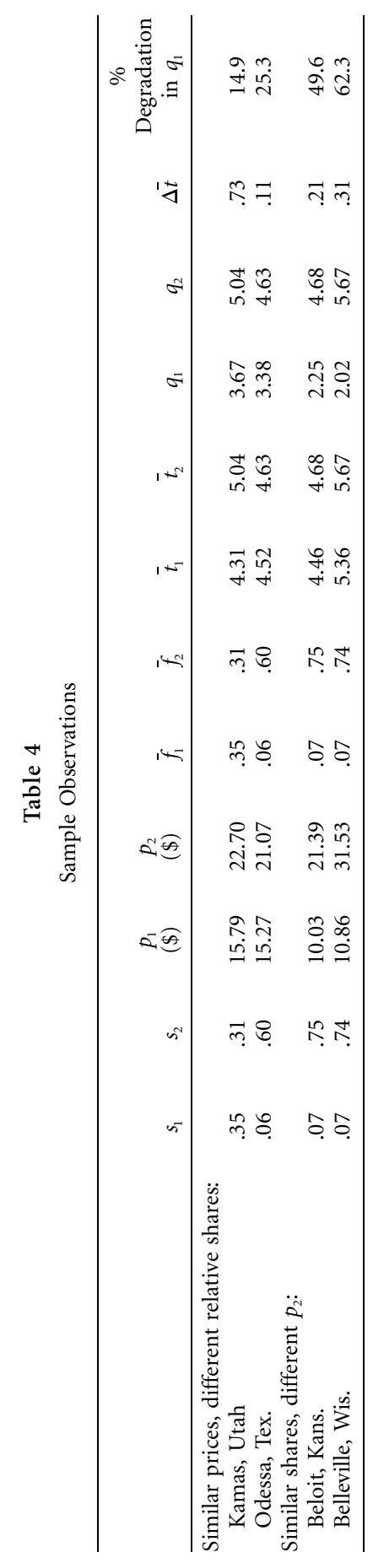


incorporate the implications of Bertrand-Nash pricing on markups to enhance the precision of estimates of consumer preferences (for example, Bresnahan 1987; Berry, Levinsohn, and Pakes 1995). In our framework, we use the implications of optimal pricing and optimal quality choice to identify the distribution of consumers' WTP for characteristics, albeit for only a single dimension of differentiation (quality). Our basic idea, however, is that there is important information in the variation in characteristics across markets that informs the shape of the distribution of preferences for those characteristics.

There are two important limitations, however, to our approach. The first is that qualities $q_{c}$ and preferences for quality $\bar{t}_{c}$ exactly solve each firm's quality choice problem in market $c$ without error. In essence, this introduces as many parameters as observations in the data, which makes our calculations of net preferences across markets mere transformations of the data rather than statistical estimates. ${ }^{31}$ We could relax this restriction, however, by pooling information across markets. It is more realistic in this case to estimate a continuous distribution of WTP for quality that is common across markets (conditional on covariates) as well as for the offered quality in each. We could also relax some of the strong assumptions on the structure of preferences and costs, and the identification requirements would be similar to those for the demand estimation literature. The disadvantage of this approach is that the computational burden is significantly larger. Not only must each firm's quality choice problem be solved for each market at each iteration in the econometric estimation, but the techniques required to do so for continuous distributions of tastes are generally more challenging than those used here. We undertake this task in Crawford and Shum (2005).

The second limitation is that the methods developed here are suited to a monopolist facing a single dimension of differentiation. We think the extension of these techniques to competition with quality choice and multiple dimensions of differentiation is quite promising, however, and discuss this in greater detail in Section 6.

\section{Results}

\subsection{Type Parameters, Quality, and Quality Degradation}

The results of our empirical procedure yield vectors of fundamental demand parameters: $\theta_{c} \equiv\left\{\bar{f}_{i c}, \bar{t}_{i c} ; i=4-n, \ldots, 3\right\}$; for each market $c=1, \ldots$, $C$. Table 5 reports the net type distributions, $\left(\bar{f}_{i}, \bar{t}_{i}\right)$, for markets offering one, two, and three goods. By construction, the share of the distribution of consumer types that purchase each good, $\bar{f}_{i}$, matches exactly the corresponding average

\footnotetext{
${ }^{31}$ Our approach provides an interesting contrast to the differentiated product demand literature in this regard. While those product demand studies also solve for each product's quality (mean utility) in each market, they restrict the distribution of consumers' preferences to be the same across markets.
} 
Table 5

Recovered Parameter Values and Implied Qualities

\begin{tabular}{|c|c|c|c|}
\hline Variable & Three-Good Markets & Two-Good Markets & One-Good Markets \\
\hline \multicolumn{4}{|c|}{ Net type distribution: } \\
\hline $\bar{f}_{3}$ & .47 & .61 & .70 \\
\hline $\bar{f}_{2}$ & .12 & .04 & $\ldots$ \\
\hline$f_{1}$ & .04 & $\cdots$ & $\cdots$ \\
\hline$f_{0}$ & .37 & .35 & .30 \\
\hline$t_{3}$ & 5.15 & 4.77 & 4.35 \\
\hline $\bar{t}_{2}$ & 4.99 & 4.65 & $\ldots$ \\
\hline $\bar{t}_{1}$ & 4.90 & $\ldots$ & . . \\
\hline \multicolumn{4}{|l|}{ Qualities: } \\
\hline$q_{3}$ & 5.15 & 4.77 & 4.35 \\
\hline$q_{2}$ & 4.43 & 2.57 & $\cdots$ \\
\hline$q_{1}$ & 3.42 & $\cdots$ & . . \\
\hline \multicolumn{4}{|c|}{ \% Degradation: } \\
\hline$\left(\bar{t}_{3}-q_{3}\right) / \bar{t}_{3}$ & .00 & .00 & .00 \\
\hline$\left(\bar{t}_{2}-q_{2}\right) / \bar{t}_{2}$ & .11 & .45 & . \\
\hline$\left(\bar{t}_{1}-q_{1}\right) / \bar{t}_{1}$ & .30 & $\cdots$ & . \\
\hline \multicolumn{4}{|c|}{ Price/quality ratio } \\
\hline$q_{3} / p_{3}$ & .20 & .21 & .23 \\
\hline$q_{2} / p_{2}$ & .21 & .21 & . . \\
\hline$q_{1} / p_{1}$ & .21 & $\ldots$ & $\ldots$ \\
\hline$N$ & 72 & 240 & 730 \\
\hline
\end{tabular}

Note. Parameters of net type distribution are obtained using the procedure in Section 4.2. Quality measures are calculated using equation (12). Percentage of degradation evaluated at cut types is defined as the marginal type just inclined to purchase that quality.

market share from Table 1. The WTP for the cut types, those consumers just willing to purchase each of the offered goods, $\bar{t}_{i}$, varies across products but is on the order of $\$ 4-\$ 5$ (in 1995 dollars) per unit of quality. There is also variation in the type distribution across systems offering different numbers of goods, with markets offering more goods being populated by households with greater tastes for cable service quality. ${ }^{32}$

\subsubsection{Quality Degradation in the Cable Television Industry}

The results presented in Table 5 and equation (12) permit us to calculate the implied quality of each offered cable service in each market. There is significant quality degradation in cable markets: offered qualities for medium- and lowquality goods are at least 11.1 percent and 30.3 percent less in three-good markets and 44.7 percent less in two-good markets than what would be provided in a competitive market offering the same number of goods. ${ }^{33}$ The larger value for

${ }^{32}$ This is an interesting result, as it confirms a primary testable implication of the Mussa and Rosen (1978) model.

${ }^{33}$ One cannot say more than that because a competitive market may not offer as many goods as would the monopolist (Spence 1975). Evaluating the welfare effects of imperfect competition on the qualities offered consumers would have to take that into account. 
two-good markets is consistent with the evidence from the raw data described in Table 3.

Two factors shape our interpretation of these findings, however. First, as discussed in Section 2.1, because we calculate the quality degradation for the lowest consumer type to purchase each good, if we have accurately specified the shape of the quality cost function, these measures are a lower bound on the actual degradation facing consumers. Calculating a measure of the quality degradation to the average consumer is difficult, however, without more information (or assumptions) about the distribution of consumer types. ${ }^{34}$ Second, limiting our ability to draw strong conclusions about quality degradation is the fact that our estimates are quite sensitive to assumptions about the curvature of the cost function used in the estimation. The Appendix describes this issue in more detail; we simply note here that while all of our results that follow are quite robust to assumptions on cost, the estimated quality degradation is not. In Crawford and Shum (2005), we generalize the empirical model used here to identify the shape of preferences and costs and draw stronger conclusions about quality degradation in cable markets.

\subsubsection{Interpreting Quality Levels}

From Table 5, the average qualities vary from a low of 2.57 to a high of 5.23 units across products and markets. To assist in the interpretation of these magnitudes, Table 6 presents a simple regression of implied qualities on the characteristics of the cable services whose quality they measure. Since cable services are bundles of programming networks, this amounts to the following regression:

$$
q_{i c}^{*}=\beta^{\prime} X_{i c}+\varepsilon_{i c}, \forall i, c,
$$

where $X_{i c}$ are the indicators of the programming offered on service $i$ in market $c$ and $\varepsilon_{i c}$ measures market-product deviations from the expected quality given $X_{i c}$. Included in this specification are dummy variables for the top 15 networks (compare Table 2), additional cable networks, and measures of broadcast network availability. If the choice of networks offered on service $i, X_{i c}$, depends on preferences for quality but not on idiosyncratic tastes for networks, the coefficient

\footnotetext{
${ }^{34} \mathrm{~A}$ very crude measure of the quality degradation to the average consumer may be obtained by assuming a uniform (within products) distribution of types and particular values (or a range of values) for the upper bound of the type distribution. If we calculate a range of upper bounds by assuming the density of consumers above the highest cut type ranges from the low to the high of the three values available for the density of consumers purchasing low- and medium-quality goods in two- and three-good markets, we find an upper bound of between 5.90 and 8.77 in three-good markets, 5.64 and 9.50 in two-good markets, and 5.36 and 9.77 in one-good markets. The implied quality degradation to the average consumer is then 29.6 percent, 12.1 percent, and 5.9-25.2 percent in three-good markets; 47.6 percent and 8.3-33.1 percent in two-good markets; and 10.1-38.3 percent in one-good markets. While capable only of a broad range of values, these average effects suggest nonnegligible quality degradation throughout the cable product line.
} 
Table 6

Interpreting Quality Measures

\begin{tabular}{lcc}
\hline Variable & Estimate & Implied Mean WTP (\$) \\
\hline WTBS & $.53(.03)$ & 2.39 \\
Discovery & $.16(.04)$ & .73 \\
ESPN & $.94(.04)$ & 4.22 \\
USA & $.33(.03)$ & 1.49 \\
CSPAN & $.08(.03)$ & .34 \\
TNT & $-.13(.04)$ & -.59 \\
Family & $.47(.03)$ & 2.13 \\
Nashville & $.31(.03)$ & 1.38 \\
Lifetime & $.02(.03)$ & .09 \\
CNN & $.21(.03)$ & .94 \\
A\&E & $.22(.03)$ & 1.01 \\
Weather & $.02(.03)$ & .10 \\
QVC & $.57(.04)$ & 2.57 \\
Learning & $.15(.04)$ & .67 \\
MTV & $.08(.03)$ & .37 \\
Other networks & $.04(.00)$ & .18 \\
\hline
\end{tabular}

Note. Coefficient estimates from regression of recovered quality levels on broadcast and cable programming variables. Reported are results for the top 15 cable networks listed in Table 2. The results are pooled across all markets and across all bundles within a market. Standard errors are in parentheses. The second column is from the authors' calculations; the estimated willingness to pay (WTP) is the product of the regression coefficient in the first column with the average (across all markets) estimated WTP for quality of the consumer just willing to purchase the high-quality bundle; $\bar{t}_{3}=4.52$.

estimates can be interpreted as the causal effects of inclusion of each network on overall service quality. ${ }^{35}$

The first column of Table 6 presents the results of this regression. ${ }^{36}$ The interpretation of the coefficient on ESPN, for example, is that adding ESPN to a cable service is estimated to increase the quality of that service by .94 "utils." The second column of Table 6 transforms this effect into the WTP for the average consumer just willing to purchase the high-quality good, that is, the average $\bar{t}_{3}$ across markets. This equals 4.51 and implies an average WTP for this consumer type for ESPN of $\$ 4.22$. The resulting estimates are generally reasonable in sign and magnitude: 12 of 15 are positive and significant, ranging from $\$ .34$ to $\$ 4.22 .{ }^{37}$ These are consistent with (and indeed more reasonable than) previous results reported by Crawford (2000). This suggests that our empirical procedure is recovering accurate measures of preferences and qualities: higher quality cable services are those with more (and more popular) cable networks.

Figure 3 orders the recovered quality measures and illustrates the results graphically. It also facilitates the interpretation of our measures of total quality and

${ }^{35}$ This assumption will be satisfied if consumers agree on an average across markets on a preference ordering for networks (for example, TNT, USA, Nickelodeon, ESPN, and so on).

${ }^{36}$ For simplicity, we present here results pooling observations across both markets and services.

${ }^{37}$ Recall that we recover net preferences, which are defined as WTP for quality less its marginal cost. As such, the reported estimates are lower bounds on the true underlying WTP. 


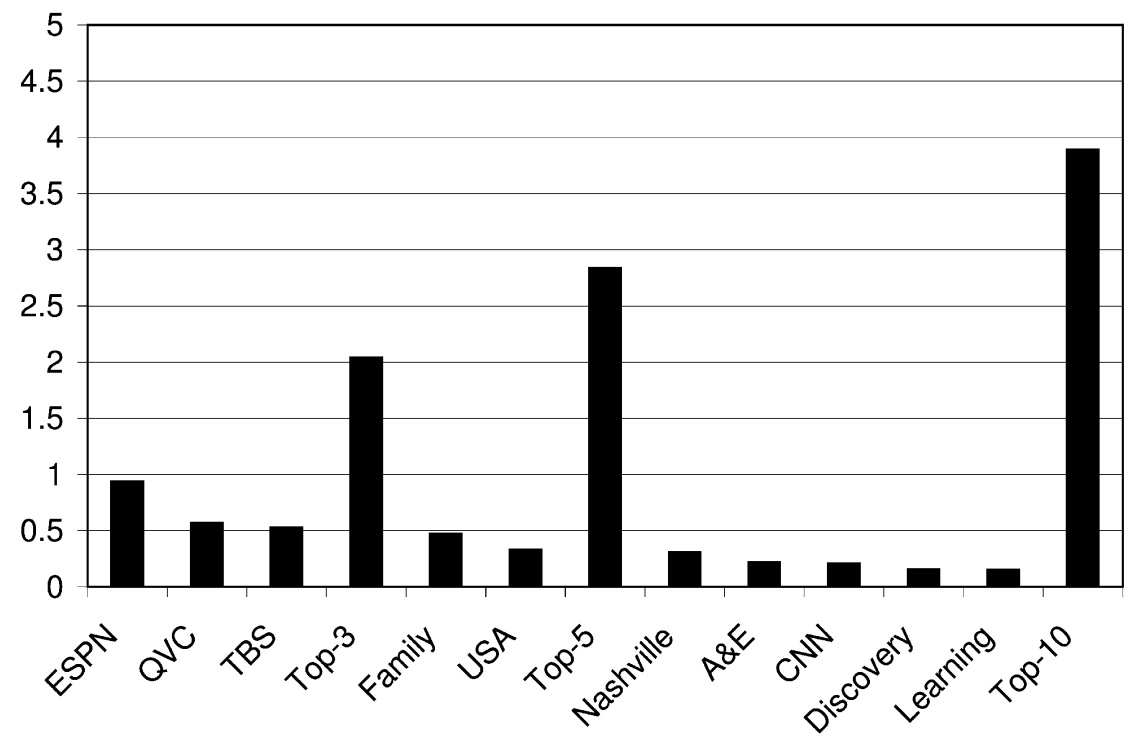

Figure 3. Recovered quality levels for top-networks results from Table 6

quality degradation presented earlier. To see this, compare Figure 3 with Figure 4, which illustrates the quality degradation results from Table 5. In particular, the regression results illustrated in Figure 3 translate the raw quality values into comparable bundles of cable networks. For example, the average quality of a low-quality good in a three-good market (3.52) is slightly less desirable than a service incorporating the 10 highest quality cable networks, as estimated in Table 6 (3.89).$^{38}$ It also translates the degree of degradation into comparable bundles of networks. For example, the average degradation of medium-quality goods in three-good markets is .54, or about the value of a single high-quality cable network like TBS or the Family Channel. At the other extreme, the average degradation for a low-quality good in a two-good market is 2.2 , or a little less than the combined value of TBS, the Family Channel, USA, the Nashville Network, A\&E, CNN, and the Discovery Channel.

\subsection{Cable Quality and the Benefits of Regulation}

As described in Section 2.2, Besanko, Donnenfeld, and White (1987, 1988) address the impact of various forms of regulation on quality offerings of a discriminating monopolist. Most relevant for our purposes are MQSs, as they were the component of the regulations imposed by the 1992 cable act that are

${ }^{38}$ This should not strike the reader as high, as even low-quality cable services in three-good markets have on average 6.5 local broadcast networks (average quality $=1.04$ ), almost 11 "other networks" (average quality $=.00$ ), and almost 12 top-40 cable networks. 


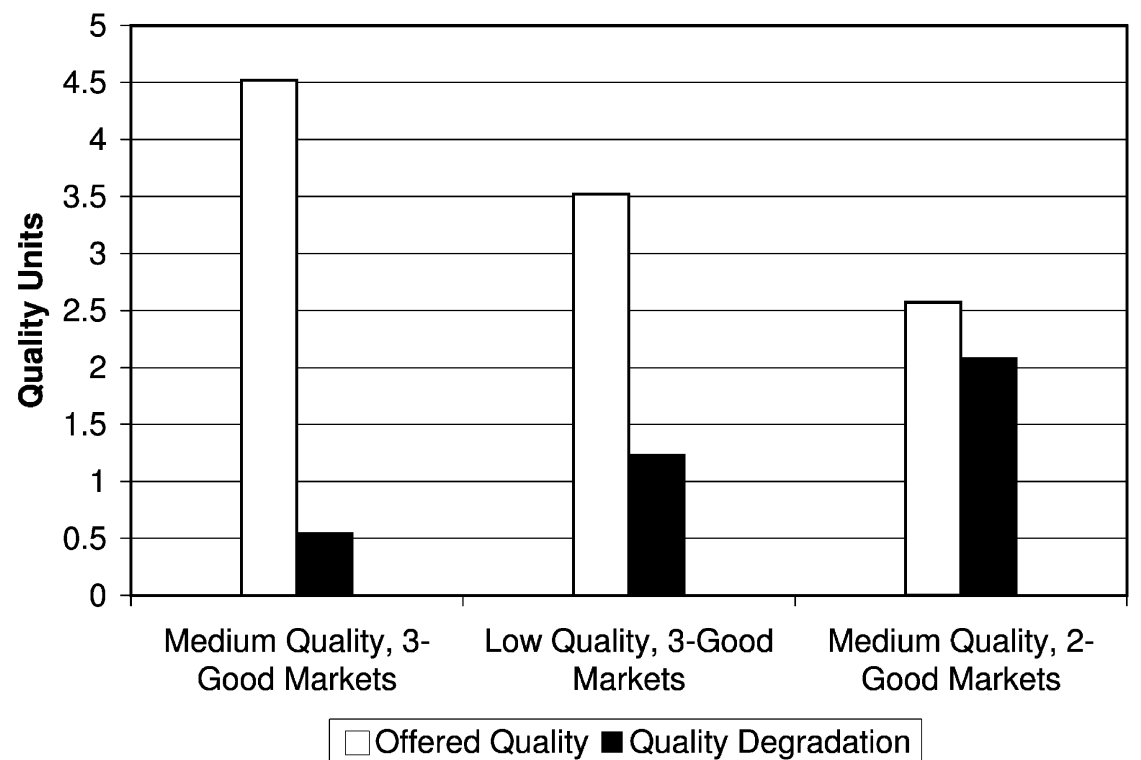

Figure 4. Quality and quality degradation by market results from Table 5

most likely to be binding in the period we study. ${ }^{39}$ Besanko, Donnenfeld, and White find that MQSs raise the monopolist's nonlinear tariff from below, increasing quality and prices for low-quality goods, lowering prices, and leaving quality unchanged for high-quality goods. ${ }^{40}$

To measure these effects, we consider reduced-form regressions of these features of cable services on characteristics of cable systems and the markets they serve. A number of variables were considered; Tables 7 and 8 present a summary of the most relevant results. ${ }^{41}$

\subsubsection{Quality and Its Degradation}

We begin by regressing recovered service qualities on a set of core system characteristics, market characteristics, and regulatory characteristics. Homes

${ }^{39}$ By contrast, price caps, while the centerpiece of the regulations imposed by the 1992 cable act, were by 1995 largely nonbinding. See Section 3 .

${ }^{40}$ The minimum-quality standard reduces the ability to distort quality downward to the low type in order to extract rents from high types. As a result, the low quality is increased (increasing its price). This makes the low-quality good more attractive to high types, which requires the monopolist to lower its price for that good. Note that while prices to low types rise (because of the higher quality), their surplus (and total surplus) generally increases.

${ }^{41}$ Variables other than the ones presented in the table but also considered included measures of system age, presence of fiber-optic cable, other demographic characteristics, and the degree of urbanization in the market. Including these variables did not change the qualitative findings presented here. 


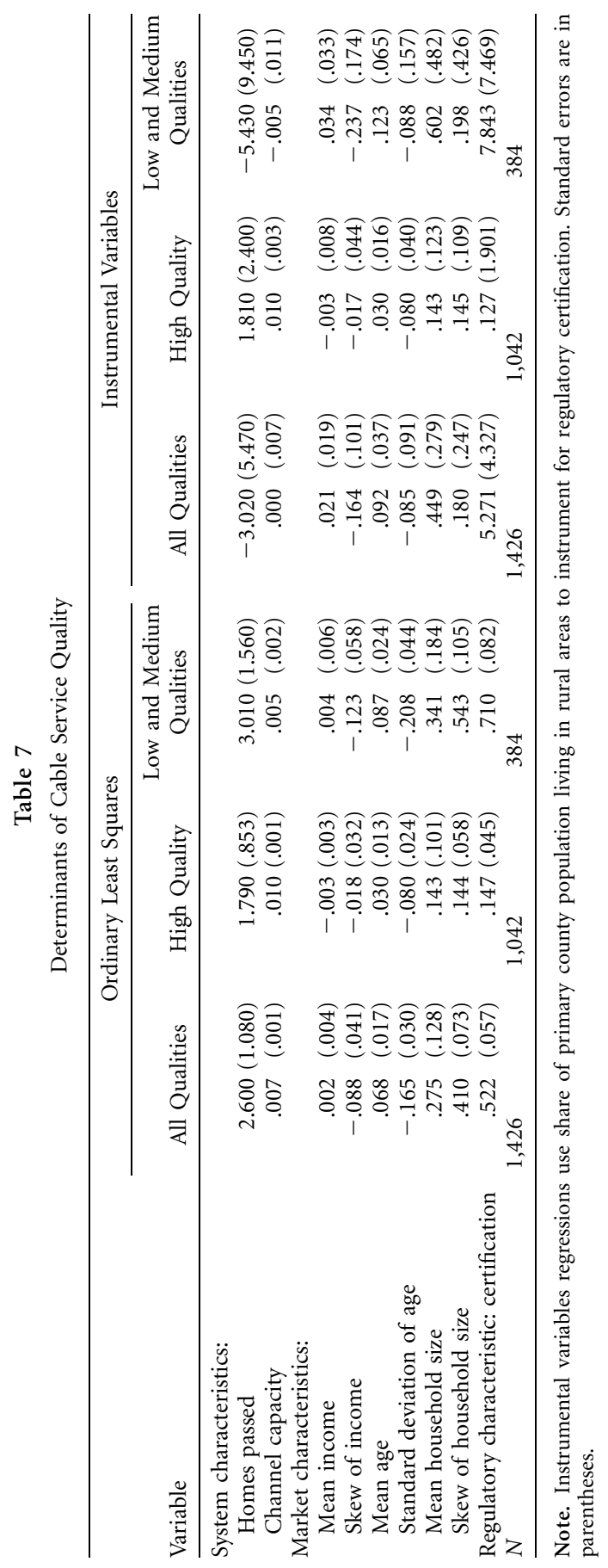




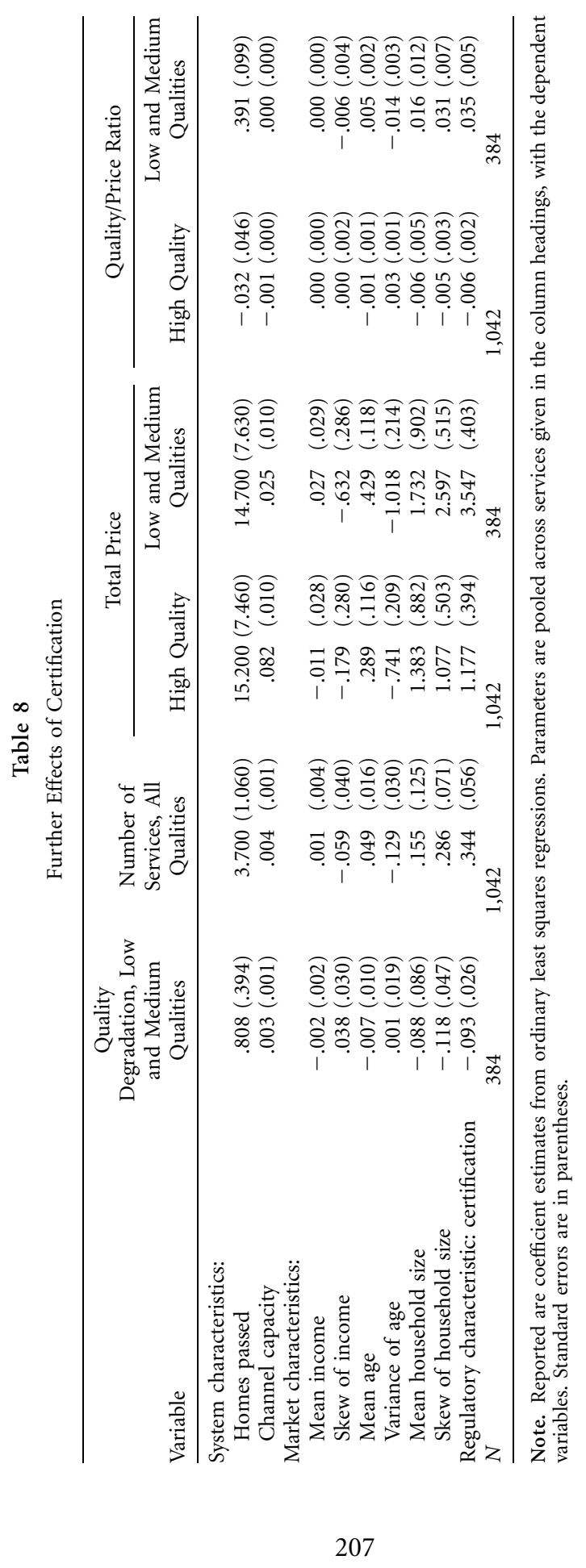


passed and channel capacity were the included system characteristics. Homes passed measures the set of households for which cable service is available in a cable market; it is a measure of local market size. This could be important, as systems in larger markets may have greater incentives to invest in higher quality services. ${ }^{42}$ Channel capacity measures the capacity of a system's technical infrastructure. Since more networks necessarily implies higher quality service, having larger channel capacity prevents capacity limitations that could hold down offered service qualities.

Features of the distribution of households within markets were the included market characteristics. To the extent household characteristics are correlated with WTP for cable service quality, the Mussa-Rosen theory has strong implications for the relationship between offered qualities and features of the distribution of these characteristics. ${ }^{43}$ The household characteristics included were income, age, and household size. Income was included because heterogeneity in WTP is often thought to be driven by heterogeneity in income. Age and household size were included because demand for cable services is known to vary in important ways with these household characteristics.

We faced a challenge, however, in parsimoniously characterizing the impact of the distribution of household characteristics on our quality measures. After considering a number of alternative specifications, we present results including the mean and skew of household income, the mean and variance of household age, and the mean and skew of household size. In all cases, we included the mean to capture the impact of increases in the location of the distribution on quality. We include the skew of income because increases in skew (controlling for the mean) induce a mean-preserving rightward shift in the distribution of income. If income is correlated with WTP for quality, this will increase incentives for quality degradation to low-quality goods. We include the skew of household size for similar reasons. We include the variance of age because there appeared to be a significant nonmonotonicity in the relationship between age distributions and qualities. Markets with relatively high shares of young people and markets with relatively high shares of old people appeared to have impacts similar to each other and different from markets with typical shares of these ages. Increasing the variance of the age distribution (controlling for the mean) induces a meanpreserving shift of the age distribution within markets into the tails.

Finally, certification to regulate prices of basic cable services served as our measure of the degree of local regulatory oversight in a system's market area. As described in Section 3.1, it measures whether the local regulatory authority had certified with the FCC to regulate the lowest quality cable service offered in the market, as permitted by the 1992 cable act, at the time the data were sampled.

The first column of Table 7 presents the estimated relationship between these

\footnotetext{
42 This phenomenon is common in media markets (Owen and Wildman 1992).

${ }^{43}$ We thank a referee for suggesting these specifications.
} 
factors and service quality. This first column pools the data across all products within markets and is meant to illuminate the broad patterns we find in our results. As expected, systems with larger market areas and greater channel capacity offer higher quality cable services. Household characteristics had very interesting impacts on offered qualities. While mean income was not estimated to influence offered qualities, increases in the skew of income tended to decrease them. Mean age was estimated to have a small positive effect on qualities, but increases in the skewness of income had a large negative effect, perhaps because of a relative lack of viewing options suitable to young or old viewers. Increases in both mean household size and share of large households controlling for mean size increased offered qualities. Finally, cable service quality is significantly larger (.52 units, equal to a top-three cable network) in markets with local regulatory oversight.

The second and third columns of Table 7 provide further insights into the relationship between market and regulatory characteristics on offered cable service quality. Of the market characteristics, the results for income are the most interesting. While the pooled results suggest that markets with more high-income households (controlling for mean income) are offered lower quality services, decomposing the effects between high- versus low- and medium-quality services shows that this effect persists only (and is much stronger) for the latter. This is exactly as predicted by the theory and suggests that the distribution of household incomes within markets is closely related to WTP for service quality.

We get similar differences in effects between service qualities for regulatory characteristics. While the pooled results in the first column suggest a strong positive relation across all services, decomposing the effect between high- versus low- and medium-quality services (if offered) yields a much stronger association at the low end of the quality spectrum. For example, the quality of the highquality services is higher by .15 , or 3.3 percent of the average quality of highquality goods, in markets with local regulatory oversight. Moreover, low- and medium-quality services are higher by .71 , or 24.4 percent of the average quality of such goods.

What of the impact of regulation on quality degradation? We consider two measures. The first is our measure of quality degradation for the existing lowand medium-quality services that are presented in Table 5 . Recall, however, that the presence of low-quality services limits the ability of the monopolist to extract rents on high-quality services. It is therefore in the firm's interest to offer lowquality services only if the profit earned from the households that buy them more than offsets the lost profits on high-quality purchasers. Another measure then of service degradation is the absence of low- and medium-quality services.

The first and second columns of Table 8 present reduced-form regressions of these measures of quality degradation on system, market, and regulatory characteristics. System variables like homes passed and channel capacity have competing effects across specifications: they appear to increase degradation where low- and medium-quality services are offered but also increase the likelihood of offering such services. Market characteristics have effects consistent with the 
quality regulations described above. Increases in the skew of income (weakly) increase quality degradation and reduce the number of offered services, while increases (decreases) in the skew (variance) of household size (age) reduce quality degradation and increase the number of offered services. Local regulatory certification, however, has a strong positive association: quality degradation is lower by .093 , or an estimated 24.1 percent of the average degradation, in certified markets, and the number of services offered is higher by .344 , or 25.1 percent of the average number of services.

\subsubsection{Endogenous Regulation}

A potential problem with these results is that regulation is unlikely to be exogenous to a system's choice of service quality. It is more likely that franchise areas choose to regulate cable systems where offered quality is low. While any such relationship would likely bias downward the estimated impact of regulation on service quality, we acknowledge the potential problem and address it by instrumenting for the certification variable in the regressions.

We consider a number of potential instruments based on likely determinants of the decision to regulate cable service. These are based on the idea that households (through their representatives in local government) make the decision to regulate their local cable television service on the basis of typical factors associated with political decisions. Features of cable service are surely important determinants of the demand for cable regulation but are poor choices for instruments, as they are likely to be correlated with unobserved elements of cable service quality. Instead, we focus on the costs associated with regulation.

As many have argued since Olson (1965), the harder government regulations are to implement, the more difficult it is to inform and organize the local population. We proxy for these costs with the share of a county's population that lives in rural areas. ${ }^{44}$ This measures the extent to which the population in a county is dispersed away from its population center. The greater this dispersion, the more difficult it is likely to be to organize an effective regulatory regime. ${ }^{45}$ Consistent with these beliefs, first-stage regressions uniformly show a strong and statistically significant negative association between rural population share and certification to regulate. ${ }^{46}$

The remaining columns of Table 7 duplicate the analysis of regulation on service quality using the rural population share as an instrument for the decision of a local franchise area to certify to regulate cable television services. As expected, instrumenting for the regulation decision increases the estimated impact of reg-

${ }^{44}$ Most cable franchise areas encompass one or a few counties. We focus on the largest county served by the system.

${ }^{45}$ A referee notes that the rural population share can also be justified as an instrument if rural populations simply have lower tastes for regulation in general.

${ }^{46}$ We also considered as cost-side instruments the size of the primary county and the share of the population in that county that rents, but these had less power and tended to produce very imprecise results. 
ulation on service quality. As shown earlier, this effect is concentrated on lowand medium-quality services: now just a 20 percent increase in the likelihood of being regulated results in an estimated increase of 1.56, or 53.8 percent, in the quality of these goods. Instrumenting also, however, greatly reduces the precision of our estimated effects, and we cannot reject the hypothesis that regulation has no effect.

The qualitative effect of instrumenting for certification in the analysis of quality degradation in Table 8 is similar to that for service quality levels: the estimated effect of certification becomes larger (in absolute value) but less precise. In what follows we therefore focus on the ordinary least squares results and take these as a lower bound of the true effects of regulation. For reference, Table A1 presents the corresponding instrumental variables results.

\subsubsection{Quality, Prices, and Regulation}

Our results so far demonstrate that local regulatory oversight is consistently associated with more and higher quality services with less degradation to consumers. These are strong results for an industry for which regulation has recently been considered an ineffective counter to the cable system's market power. Is cable regulation then beneficial to consumers? Does it translate into consumer welfare benefits? While we cannot answer this question directly, we present here what our results suggest about this important public policy issue.

To test these implications, we ran reduced-form regressions of cable prices and quality/price ratios on the same characteristics of cable systems and the markets they serve. Table 8 presents the results. Several interesting patterns emerge. First, prices are demonstrated to be higher in markets subject to local regulatory oversight, with a higher certification coefficient for low- and mediumquality goods (3.547) relative to high-quality goods (1.177). As their nominal purpose was to reduce prices, this has been taken as evidence that the regulations imposed by the 1992 cable act did not work (Crawford 2000; Hazlett and Spitzer 1997). In practice, however, the regulations introduced by the cable act capped prices on a per-channel basis, making the quality/price ratio the relevant unit of analysis. Despite the higher prices, quality/price ratios are substantially higher for low- and medium-quality goods (.035, or 16.6 percent) and marginally lower (by .006, or 2.1 percent) for high-quality goods. Note these effects are exactly what are predicted by Besanko, Donnenfeld, and White (1988) and suggest that consumers may well have benefited from the regulations introduced by the 1992 cable act, not because of lower prices from the imposition of price caps but because of significantly increased quality for a slightly higher price from the introduction of MQSs. While a detailed welfare analysis is beyond the scope of this study, this suggests at worst a distributional impact from the regulations, with low-taste (presumably low-income) consumers benefiting at the expense of high-taste (high-income) consumers. 


\section{Conclusions}

In this paper, we measure the extent of quality degradation in cable television markets. Using an empirical framework taken from the standard theoretical nonlinear pricing models, we recover the quality levels of the offered cable services as well as features of the distribution of consumer preferences. These measures allow us to directly quantify the degree of quality degradation in cable markets. We find lower bounds of quality degradation ranging from 11 to 45 percent of observed service qualities. Furthermore, local regulatory oversight is associated with significantly higher quality and lower quality degradation.

Several extensions of the existing analysis are suggested. On institutional grounds, by developing techniques to accurately measure product quality in the cable television industry, the failure of the early-1990s cable regulatory experience seems not so obvious. In light of continued consumer frustration over increasing cable prices and consequent calls for reregulation, if not of prices then perhaps of product offerings (see, for example, Consumers Union 2003). Our results suggest both a more detailed analysis of the effects of past regulations and careful consideration of the consequences to both prices and qualities of new regulatory solutions.

On methodological grounds, while the existing specification can recover quite flexibly the distribution of consumer tastes in each cable market, it does not admit controlling for observed or unobserved heterogeneity in cost and demand. Extending it would require pooling information about preferences and costs across markets but would permit greater confidence in the estimated effects of endogenous quality as well as measurement of the consumer and social welfare consequences of endogenous quality choice. Each of these extensions is the topic of ongoing research (Crawford and Shum 2005).

Furthermore, while the data and industry we study necessitate the analysis of the monopoly problem, we think the lessons we learn here might apply much more broadly. First, research into competition with prices and qualities (so-called competitive nonlinear pricing) finds that rivalry generally reduces distortions in both dimensions (Stole 2002). As such, our findings provide evidence of how bad the problem can be. More important, however, is that the methodological approach we take here-based on the screening literature analyzing nonlinear pricing - has enormous potential to generalize both to larger, multidimensional, quality (or characteristic) spaces as well as to competitive settings. Thus it has the potential to solve the long-standing problem of endogenous product characteristics that is acknowledged in many recent empirical analyses of oligopolistic markets (see, for example, Berry, Levinsohn, and Pakes 1995, p. 854; Nevo 2001, p. 322; Petrin 2003, p. 24). While there are significant computational burdens involved in both cases (compare Rochet and Stole 2002; Stole 2002), the approach we take here of allowing continuous distributions of consumer preferences but discrete goods offered by firms may be both empirically realistic and tractably extensible in one or both dimensions. 


\section{Appendix A}

\section{Sensitivity Analysis}

The results presented in this paper rely on some fairly strong assumptions about the nature of preferences and costs. In this Appendix, we consider the sensitivity of our results to these assumptions.

As the form of the surplus function critically determines the magnitude of the first-best qualities and, hence, the extent of degradation, we focus on the sensitivity of our results to the assumed parametric form of the cost function, which is one component of the surplus function. In particular, we consider the consequences to our results if costs are of the form

$$
C\left(q_{i}\right)=\frac{1}{\rho+1} q_{i}^{\rho+1}
$$

Marginal costs for quality are $C^{\prime}\left(q_{i}\right)=q_{i}^{\rho}$, which imply that $\rho$ measures the rate at which marginal costs for quality increase with quality. In the baseline specifications reported above, we chose $\rho=1$, which implies that marginal costs increase linearly with $q_{i}$.

Unfortunately, as we are just identified in each market, we cannot separately identify $\rho$ in the data. ${ }^{47}$ Instead, we solve the model for various values of $\rho$ and assess the robustness of our conclusions to these assumptions.

Tables A2-A4 present the results of these tests. Table A2 presents parameter values and implied qualities consistent with different values of the cost curvature parameter $\rho$. Note that $\rho=1$ corresponds to the baseline results presented in Tables 5-8. As is evident, the cut points of the type distribution, implied qualities, and quality degradation are each sensitive to assumptions on $\rho$. In particular, as the cost function gets steeper, values for cut types increase, qualities decrease, and degradation increases.

Nevertheless, while each of the cut types, $\bar{t}$, and quality, $q$, are sensitive to $\rho$, the utility they provide, measured by their product, $\bar{t} \times q$, is not. This can be seen in Tables A3 and A4. Table A3 duplicates the quality regressions relating quality of each offered service to the networks provided on each. While the parameter estimates vary across the columns, the mean WTP for each network $\left(t_{3} \times \beta_{k}\right)$ is quite stable. Similar results obtain for the impact of regulation on cable service quality presented in Table A4. While the parameter estimates vary with $\rho$, the WTP equivalent of each covariate is stable across $\rho$. Similar effects obtain for degradation and price/quality ratios. As a result, we take caution in making strong claims about magnitudes that are sensitive to $\rho$ (for example, the percent quality degradation) but feel confident in our conclusions about magnitudes that appear robust to variation in $\rho$.

${ }^{47}$ Crawford and Shum (2005) presents a more general model that does estimate the cost (and preference) structure using a more recent cable dataset. 


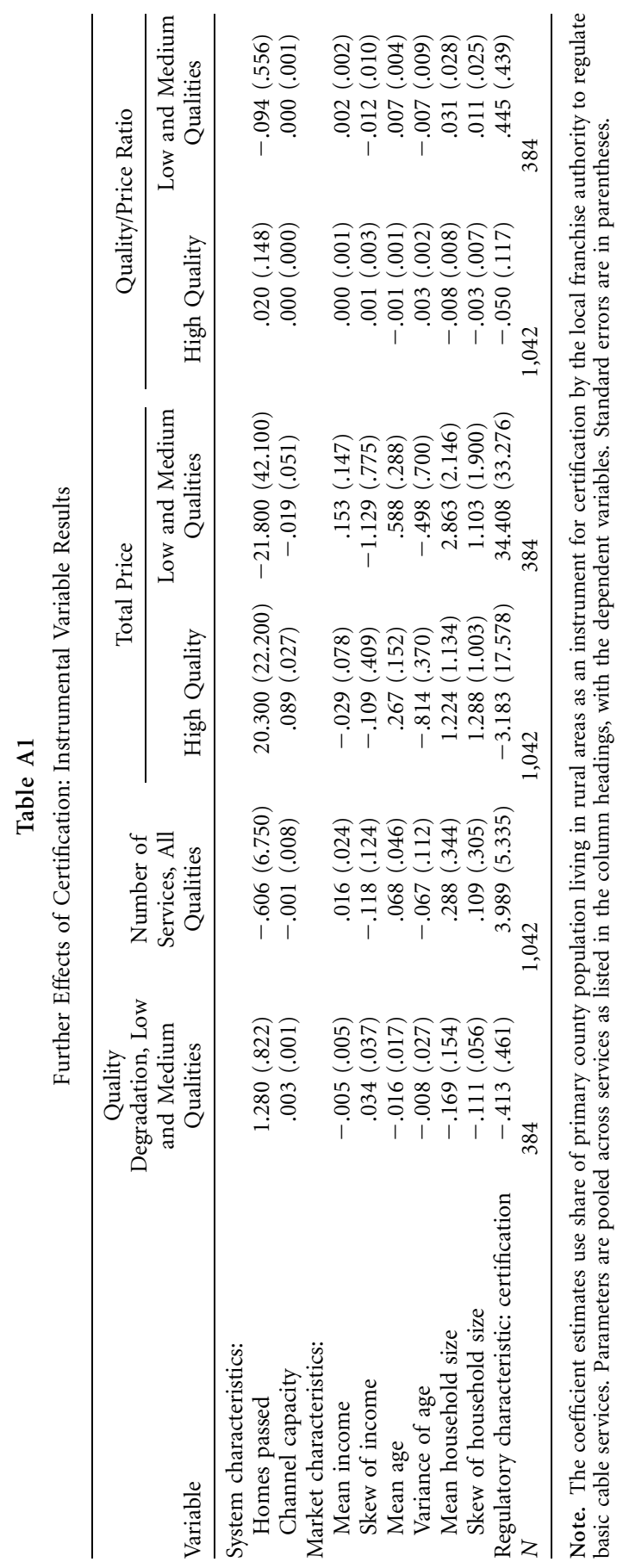


Table A2

Parameters and Implied Qualities: Sensitivity Analysis

\begin{tabular}{|c|c|c|c|c|c|c|c|c|c|}
\hline \multirow[b]{2}{*}{ Variable } & \multicolumn{3}{|c|}{$\rho=1$} & \multicolumn{3}{|c|}{$\rho=1.5$} & \multicolumn{3}{|c|}{$\rho=2$} \\
\hline & $\begin{array}{l}\text { Three } \\
\text { Good }\end{array}$ & $\begin{array}{l}\text { Two } \\
\text { Good }\end{array}$ & $\begin{array}{l}\text { One } \\
\text { Good }\end{array}$ & $\begin{array}{l}\text { Three } \\
\text { Good }\end{array}$ & $\begin{array}{l}\text { Two } \\
\text { Good }\end{array}$ & $\begin{array}{l}\text { One } \\
\text { Good }\end{array}$ & $\begin{array}{l}\text { Three } \\
\text { Good }\end{array}$ & $\begin{array}{l}\text { Two } \\
\text { Good }\end{array}$ & $\begin{array}{l}\text { One } \\
\text { Good }\end{array}$ \\
\hline \multicolumn{10}{|l|}{ Types: } \\
\hline$t_{3}$ & 5.15 & 4.77 & 4.35 & 7.23 & 6.55 & 5.85 & 9.10 & 8.02 & 7.12 \\
\hline$\vec{t}_{2}$ & 4.99 & 4.65 & 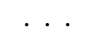 & 6.90 & 6.33 & & 8.57 & 7.74 & $\cdots$ \\
\hline $\bar{t}_{1}$ & 4.90 & $\ldots$ & $\cdots$ & 6.73 & $\cdots$ & & 8.31 & $\cdots$ & $\cdots$ \\
\hline \multicolumn{10}{|l|}{ Quality: } \\
\hline$q_{3}$ & 5.15 & 4.77 & 4.35 & 3.73 & 3.49 & 3.24 & 3.01 & 2.82 & 2.66 \\
\hline$q_{2}$ & 4.43 & 2.57 & $\ldots$ & 3.22 & 1.89 & $\ldots$ & 2.60 & 1.57 & $\ldots$ \\
\hline$q_{1}$ & 3.42 & $\ldots$ & $\ldots$ & 2.49 & $\ldots$ & $\ldots$ & 2.01 & $\ldots$ & $\ldots$ \\
\hline \multicolumn{10}{|l|}{ Degradation: } \\
\hline$\left(\bar{t}_{3}-q_{3}\right) / \bar{t}_{3}$ & .00 & .00 & .00 & .00 & .00 & .00 & .00 & .00 & .00 \\
\hline$\left(t_{2}-q_{2}\right) / t_{2}$ & .11 & .45 & $\ldots$ & .53 & .70 & . & .69 & .79 & $\ldots$ \\
\hline$\left(t_{1}-q_{1}\right) / t_{1}$ & .30 & & & .63 & & & .76 & & \\
\hline$N$ & 72 & 240 & 730 & 72 & 240 & 730 & 72 & 240 & 730 \\
\hline
\end{tabular}

Note. Parameters of net type distribution are obtained using the procedure in Section 4.2. Qualities are calculated using these values and equation (12). Percentage of degradation was evaluated at cut types, which are defined as the marginal types just inclined to purchase that quality.

Table A3

Interpreting Quality Measures: Sensitivity Analysis

\begin{tabular}{|c|c|c|c|c|c|c|}
\hline \multirow[b]{2}{*}{ Variable } & \multicolumn{2}{|c|}{$\rho=1$} & \multicolumn{2}{|c|}{$\rho=1.5$} & \multicolumn{2}{|c|}{$\rho=2$} \\
\hline & Estimate & $\begin{array}{c}\text { Implied } \\
\text { Mean } \\
\text { WTP }\end{array}$ & Estimate & $\begin{array}{c}\text { Implied } \\
\text { Mean } \\
\text { WTP }\end{array}$ & Estimate & $\begin{array}{l}\text { Implied } \\
\text { Mean } \\
\text { WTP }\end{array}$ \\
\hline WTBS & $.57(.03)$ & 2.58 & $.43(.02)$ & 2.60 & $.28(.02)$ & 2.08 \\
\hline Discovery & $.14(.03)$ & .62 & $.09(.02)$ & .57 & $.03(.02)$ & .22 \\
\hline ESPN & $.94(.04)$ & 4.25 & $.70(.03)$ & 4.29 & $.49(.02)$ & 3.66 \\
\hline USA & $.28(.03)$ & 1.25 & $.21(.02)$ & 1.31 & $.11(.02)$ & .79 \\
\hline CSPAN & $.08(.03)$ & .35 & $.05(.02)$ & .31 & $.03(.02)$ & .20 \\
\hline TNT & $-.03(.03)$ & -.13 & $-.03(.02)$ & -.18 & $.03(.02)$ & .21 \\
\hline Family & $.38(.03)$ & 1.71 & $.27(.02)$ & 1.67 & $.22(.02)$ & 1.64 \\
\hline Nashville & $.25(.03)$ & 1.14 & $.18(.02)$ & 1.08 & $.12(.02)$ & .87 \\
\hline Lifetime & $.04(.03)$ & .16 & $.02(.02)$ & .15 & $-.01(.02)$ & -.10 \\
\hline $\mathrm{CNN}$ & $.15(.03)$ & .66 & $.11(.02)$ & .68 & $.03(.02)$ & .19 \\
\hline$A \& E$ & $.24(.03)$ & 1.06 & $.17(.02)$ & 1.06 & $.13(.02)$ & .96 \\
\hline Weather & $-.01(.03)$ & -.02 & $-.01(.02)$ & -.05 & $-.04(.02)$ & -.30 \\
\hline QVC & $.56(.03)$ & 2.51 & $.40(.02)$ & 2.44 & $.40(.02)$ & 2.96 \\
\hline Learning & $.09(.03)$ & .42 & $.05(.02)$ & .32 & $.07(.02)$ & .55 \\
\hline MTV & $.18(.03)$ & .81 & $.13(.02)$ & .77 & $.11(.02)$ & .84 \\
\hline Other networks & $.04(.00)$ & .20 & $.03(.00)$ & .18 & $.03(.00)$ & .19 \\
\hline
\end{tabular}

Note. Coefficient estimates are from regressions of recovered quality levels on broadcast and cable programming variables. Reported are results for the top 15 cable networks listed in Table 2. Data are pooled across all markets and across all bundles within a market. Standard errors are in parentheses. Implied willingness to pay (WTP) values are from the authors' calculations. The estimated WTP is the product of the regression coefficient in the estimate column and the average (across all markets) estimated WTP for quality of the consumer just willing to purchase the high-quality bundle. 


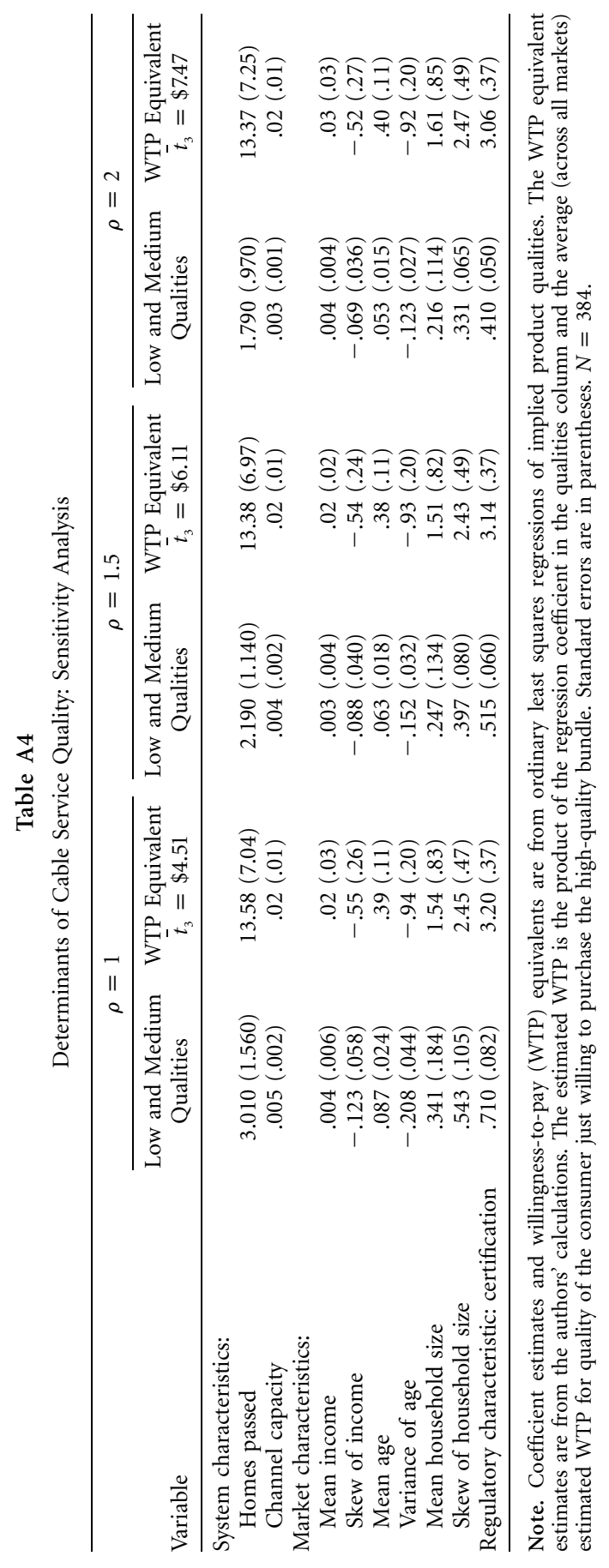




\section{References}

Berry, Steven T. 1994. Estimating Discrete-Choice Models of Product Differentiation. RAND Journal of Economics 25:242-62.

Berry, Steven, James Levinsohn, and Ariel Pakes. 1995. Automobile Prices in Market Equilibrium. Econometrica 63:841-90.

Besanko, David, Shabtai Donnenfeld, and Lawrence J. White. 1987. Monopoly and Quality Distortion: Effects and Remedies. Quarterly Journal of Economics 102:743-67.

- 1988. The Multiproduct Firm, Quality Choice, and Regulation. Journal of Industrial Economics 36:411-29.

Bousquet, Alain, and Marc Ivaldi. 1997. Optimal Pricing of Telephone Usage: An Econometric Implementation. Information Economics and Policy 9:219-39.

Bresnahan, Timothy F. 1987. Competition and Collusion in the American Auto Industry: The 1955 Price War. Journal of Industrial Economics 35:457-82.

1989. Empirical Studies of Industries with Market Power. Pp. 1011-58 in Handbook of Industrial Organization, edited by Richard Schmalensee and Robert D. Willig. Vol. 2. Amsterdam: North-Holland.

Clerides, Sofronis K. 2002. Book Value: Intertemporal Pricing and Quality Discrimination in the U.S. Market for Books. International Journal of Industrial Organization 20: 1385-1408.

Cohen, Andrew. 2001. Package Size and Price Discrimination: Evidence from Paper Towels. Unpublished manuscript. University of Virginia, Department of Economics.

Consumer Reports. 2002. Deregulated. July, pp. 30-35.

Consumers Union. 2003. FCC Report Shows Cable Rates Skyrocketing; Group Calls on Congress to Allow Consumers to Buy Programming on an a la Carte Basis. July 8.

Crandall, Robert W., and Harold Furchtgott-Roth. 1996. Cable TV: Regulation or Competition? Washington, D.C.: Brookings Institution.

Crawford, Gregory. 2000. The Impact of the 1992 Cable Act on Household Demand and Welfare. RAND Journal of Economics 31:422-49.

- 2004. The Discriminatory Incentives to Bundle in the Cable Television Industry. Unpublished manuscript. University of Arizona, Department of Economics.

Crawford, Gregory S., and Matthew Shum. 2005. Empirical Modeling of Endogenous Quality Choice: The Case of Cable Television. Unpublished manuscript. University of Arizona, Department of Economics.

Dixit, Avinash K., and Joseph E. Stiglitz. 1977. Monopolistic Competition and Optimum Product Diversity. American Economic Review 67:297-308.

FCC (Federal Communications Commission). 1997. 1996 Report on Cable Industry Prices. http://www.fcc.gov/Bureaus/Cable/Reports/fcc96499.txt.

- 2001. Annual Assessment of the Status of Competition in the Market for the Delivery of Video Programming: Seventh Annual Report. http://www.fcc.gov/Bureaus/ Cable/Reports/fcc01001.pdf.

- 2003. Report on Cable Industry Prices. http://hraunfoss.fcc.gov/edocs_public/ attachmatch/FCC-03-136A1.pdf.

—. 2005a. Report on Cable Industry Prices. http://hraunfoss.fcc.gov/edocs_public/ attachmatch/FCC-05-12A1.pdf.

2005b. Annual Assessment of the Status of Competition in the Market for the Delivery of Video Programming: Eleventh Annual Report. http://hraunfoss.fcc.gov/ edocs_public/attachmatch/FCC-05-13A1.pdf. 
Fowler, Bob. 2000. Some Anderson County, Tenn., Cable Customers Want Fewer Channels, Lower Rates. Knoxville News-Sentinel, November 19.

GAO (General Accounting Office). 1991. Telecommunications: 1991 Survey of Cable Television Rates and Services. GAO/RCED-91-195. Washington, D.C.: Government Printing Office.

Hazlett, Thomas W., and Matthew L. Spitzer. 1997. Public Policy toward Cable Television: The Economics of Rate Controls. Cambridge, Mass.: MIT Press.

Horn, Patricia. 2001. Prices Tend to Rise as Competition Lags for Cable TV. Philadelphia Inquirer, June 3.

Johnson, Leland L. 1994. Toward Competition in Cable Television. Cambridge, Mass.: MIT Press.

Kagan World Media. 2002. Broadband Cable Financial Databook, 2002. http:// www.ncta.com/.

- 2004. Cable Program Investor. March 15.

Laffont, Jean-Jacques, and Jean Tirole. 1993. A Theory of Incentives in Procurement and Regulation. Cambridge, Mass.: MIT Press.

Leslie, Phillip. 2004. Price Discrimination in Broadway Theatre. RAND Journal of Economics 35:520-41.

Maskin, Eric, and John Riley. 1984. Monopoly with Incomplete Information. RAND Journal of Economics 15:171-96.

Mayo, John W., and Yasuji Otsuka. 1991. Demand, Pricing, and Regulation: Evidence from the Cable TV Industry. RAND Journal of Economics 22:396-410.

McConnell, Bill. 2003. FCC Report Sparks Cable-Rates Debate. Broadcasting and Cable, July 9.

McManus, Brian. Forthcoming. Nonlinear Pricing in an Oligopoly Market: The Case of Specialty Coffee. Rand Journal of Economics.

Miravete, Eugenio J. 2002. Estimating Demand for Local Telephone Service with Asymmetric Information and Optional Calling Plans. Review of Economic Studies 69:943-71.

Miravete, Eugenio J., and Lars-Hendrik Röller. 2003. Competitive Nonlinear Pricing in Duopoly Equilibrium: The Early U.S. Cellular Telephone Industry. CEPR Discussion Paper No. 4069. Centre for Economic Policy Research, London.

Mussa, Michael, and Sherwin Rosen. 1978. Monopoly and Product Quality. Journal of Economic Theory 18:301-17.

Nevo, Aviv. 2001. Measuring Market Power in the Ready-To-Eat Cereal Industry. Econometrica 69:307-42.

Olson, Mancur. 1965. The Logic of Collective Action. Cambridge, Mass.: Harvard University Press.

Owen, Bruce M., and Steven S. Wildman. 1992. Video Economics. Cambridge, Mass.: Harvard University Press.

Petrin, Amil. 2003. Quantifying the Benefits of New Products: The Case of the Minivan. Journal of Political Economy 110:705-29.

Rochet, Jean-Charles, and Philippe Choné. 1998. Ironing, Sweeping, and Multidimensional Screening. Econometrica 66:783-826.

Rochet, Jean-Charles, and Lars Stole. 2003. The Economics of Multidimensional Screening. Pp. 150-97 in vol. 1 of Advances in Economics and Econometrics, edited by Mathias Dewatripont, Lars Peter Hansen, and Stephen J. Turnovsky. Cambridge and New York: Cambridge University Press. 
. 2002. Nonlinear Pricing with Random Participation. Review of Economic Studies 69:277-311.

Rubinovitz, Robert N. 1993. Market Power and Price Increases for Basic Cable Service since Deregulation. RAND Journal of Economics 24:1-18.

Spence, A. Michael. 1975. Monopoly, Quality, and Regulation. Bell Journal of Economics 6:417-29.

- 1980. Multi-product Quantity-Dependent Prices and Profitability Constraints. Review of Economic Studies 47:821-41.

Stole, Lars. 2007. Price Discrimination and Competition. Chap. 8 in vol. 3 of Handbook of Industrial Organization, edited by Mark Armstrong and Robert Porter. NorthHolland: Elsevier.

U.S. Census Bureau. 1994. County and City Data Book. Bernan Associates, Economic Data from U.S. Census Bureau on CD-Rom.

Verboven, Frank. 2002. Quality-based Price Discrimination and Tax Incidence: Evidence from Gasoline and Diesel Cars. RAND Journal of Economics 33:275-97.

Warren, Albert, ed. 1996. Television and Cable Factbook: The Authoritative Reference for the Television, Cable, and Electronics Industries.. Washington, D.C.: Warren Publishing. Wilson, Robert. 1993. Nonlinear Pricing. Cambridge: Oxford University Press.

Wolak, F. 1994. Estimating Regulated Firm Production Functions with Private Information: An Application to California Water Utilities. Annales d'Economie et de Statistique 34:13-69. 